2013

\title{
Psychosocial interventions for patients
}

\section{with head and neck cancer}

Semple, C

http://hdl.handle.net/10026.1/3146

10.1002/14651858.cd009441.pub2

Cochrane Database of Systematic Reviews

Wiley

All content in PEARL is protected by copyright law. Author manuscripts are made available in accordance with publisher policies. Please cite only the published version using the details provided on the item record or document. In the absence of an open licence (e.g. Creative Commons), permissions for further reuse of content should be sought from the publisher or author. 


\section{Psychosocial interventions for patients with head and neck cancer (Review)}

Semple C, Parahoo K, Norman A, McCaughan E, Humphris G, Mills M

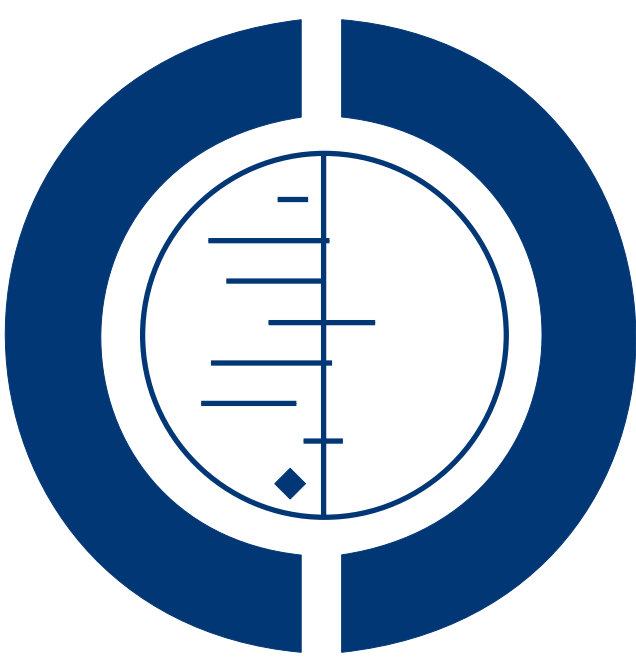

\section{THE COCHRANE COLLABORATION $^{\circledR}$}

This is a reprint of a Cochrane review, prepared and maintained by The Cochrane Collaboration and published in The Cochrane Library 2013, Issue 7

http://www.thecochranelibrary.com

\section{WILEY}

Psychosocial interventions for patients with head and neck cancer (Review)

Copyright $\odot 2013$ The Cochrane Collaboration. Published by John Wiley \& Sons, Ltd. 
TABLE OF CONTENTS

HEADER . . . . . . . . . . . . . . . . . . . . . . . . . . . . . . . . . . . . . . . . . . . . .

ABSTRACT . . . . . . . . . . . . . . . . . . . . . . . . . . . . . . . . . . . . . . . . . . . . . . 1

PLAIN LANGUAGE SUMMARY . . . . . . . . . . . . . . . . . . . . . . . . . . . . . . . . . . . . . . . . . . . . .

BACKGROUND . . . . . . . . . . . . . . . . . . . . . . . . . . . . . . . . . . . . . 3

OBJECTIVES . . . . . . . . . . . . . . . . . . . . . . . . . . . . . . . . . . . . . . . . . . . . . . . . . . .

METHODS . . . . . . . . . . . . . . . . . . . . . . . . . . . . . . . . . . . . . . 44

RESULTS . . . . . . . . . . . . . . . . . . . . . . . . . . . . . . . . . . . . . . .

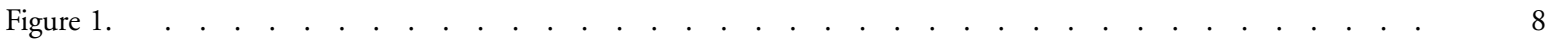

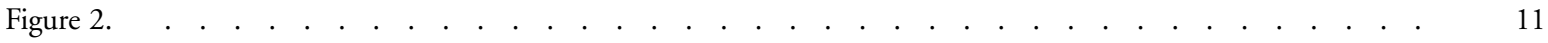

Figure 3. . . . . . . . . . . . . . . . . . . . . . . . . . . . . . . . . . . . . . 12

Figure 4. . . . . . . . . . . . . . . . . . . . . . . . . . . . . . . . . 14

Figure 5. . . . . . . . . . . . . . . . . . . . . . . . . . . . . . . . . . . . . . . . . . . 14

Figure 6. . . . . . . . . . . . . . . . . . . . . . . . . . . . . . . . . . . . . . .

DISCUSSION . . . . . . . . . . . . . . . . . . . . . . . . . . . . . . . . . . . . . . . . . . . . .

AUTHORS' CONCLUSIONS . . . . . . . . . . . . . . . . . . . . . . . . . . . . . . . . . . . . .

ACKNOWLEDGEMENTS . . . . . . . . . . . . . . . . . . . . . . . . . . . . . . . . . . . . . . . . . . . . 19

REFERENCES . . . . . . . . . . . . . . . . . . . . . . . . . . . . . . . . . . . . . . 20

CHARACTERISTICS OF STUDIES . . . . . . . . . . . . . . . . . . . . . . . . . . . . . . . . . . . . . . .

DATA AND ANALYSES . . . . . . . . . . . . . . . . . . . . . . . . . . . . . . . . . . . . . . . . . .

Analysis 1.1. Comparison 1 Intervention versus control, Outcome 1 EORTC QLQ C30 - Physical function (medium-term follow-up). . . . . . . . . . . . . . . . . . . . . . . . . . . . . . . . . . . . . . . . . . . . . . . . 37

Analysis 1.2. Comparison 1 Intervention versus control, Outcome 2 EORTC QLQ C30 - Emotional function (mediumterm follow-up). . . . . . . . . . . . . . . . . . . . . . . . . . . . . . . . . . . . 38

Analysis 1.3. Comparison 1 Intervention versus control, Outcome 3 EORTC QLQ C30 - Social function (medium-term follow-up).

Analysis 1.4. Comparison 1 Intervention versus control, Outcome 4 EORTC QLQ C30 - Role function (medium-term follow-up).

Analysis 1.5. Comparison 1 Intervention versus control, Outcome 5 EORTC QLQ C30 - Global quality of life (mediumterm follow-up).

Analysis 1.6. Comparison 1 Intervention versus control, Outcome 6 EORTC QLQ C30 - Cognitive function (mediumterm follow-up).

Analysis 1.7. Comparison 1 Intervention versus control, Outcome 7 EORTC QLQ C30 - Cognitive function (end of intervention).

Analysis 1.8. Comparison 1 Intervention versus control, Outcome 8 EORTC QLQ C30 - Physical function (end of intervention).

Analysis 1.9. Comparison 1 Intervention versus control, Outcome 9 EORTC QLQ C30 - Emotional function (end of intervention).

Analysis 1.10. Comparison 1 Intervention versus control, Outcome 10 EORTC QLQ C30 - Social function (end of intervention).

Analysis 1.11. Comparison 1 Intervention versus control, Outcome 11 EORTC QLQ C30 - Role function (end of intervention).

Analysis 1.12. Comparison 1 Intervention versus control, Outcome 12 EORTC QLQ C30 - Global quality of life (end of intervention).

Analysis 1.13. Comparison 1 Intervention versus control, Outcome 13 Anxiety (medium-term follow-up 3 to 4 months).

Analysis 1.14. Comparison 1 Intervention versus control, Outcome 14 Anxiety (end of intervention).

Analysis 1.15. Comparison 1 Intervention versus control, Outcome 15 Depression (medium term follow-up 3 to 6 months). . . . . . . . . . . . . . . . . . . . . . . . . . . . . . . . . . 51

Analysis 1.16. Comparison 1 Intervention versus control, Outcome 16 Depression (end of intervention). $\quad . \quad$. . $\quad$. 52

APPENDICES . . . . . . . . . . . . . . . . . . . . . . . . . . . . . . . . . . . 52

CONTRIBUTIONS OF AUTHORS . . . . . . . . . . . . . . . . . . . . . . . . . . . . . . . . . . . . . . . . . . . . .

DECLARATIONS OF INTEREST . . . . . . . . . . . . . . . . . . . . . . . . . . . . . . . . . . . . . . . . . . . . .

Psychosocial interventions for patients with head and neck cancer (Review)

Copyright @ 2013 The Cochrane Collaboration. Published by John Wiley \& Sons, Ltd. 
SOURCES OF SUPPORT . . . . . . . . . . . . . . . . . . . . . . . . . . . . . . . . . 56

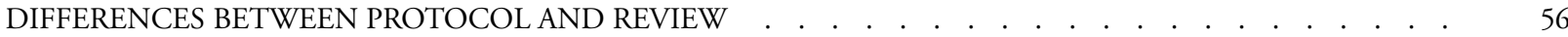




\title{
[Intervention Review]
}

\section{Psychosocial interventions for patients with head and neck cancer}

\author{
Cherith Semple ${ }^{1}$, Kader Parahoo ${ }^{2}$, Alyson Norman ${ }^{3}$, Eilis McCaughan $^{2}$, Gerry Humphris $^{4}$, Moyra Mills ${ }^{5}$ \\ ${ }^{1}$ Cancer Services, South Eastern Health \& Social Care Trust, Belfast, UK. ${ }^{2}$ Institute of Nursing and Health Research, University of \\ Ulster, Coleraine, UK. ${ }^{3}$ Psychology, University of Plymouth, Plymouth, UK. ${ }^{4}$ School of Medicine, University of St. Andrews, Fife, \\ UK. ${ }^{5}$ Northern Health and Social Care Trust, Antrim, UK \\ Contact address: Cherith Semple, Cancer Services, South Eastern Health \& Social Care Trust, Upper Newtownards Road, Belfast, \\ Northern Ireland, BT16 1RH, UK. cherith.semple@setrust.hscni.net.
}

Editorial group: Cochrane Ear, Nose and Throat Disorders Group.

Publication status and date: New, published in Issue 7, 2013.

Review content assessed as up-to-date: 17 December 2012.

Citation: Semple C, Parahoo K, Norman A, McCaughan E, Humphris G, Mills M. Psychosocial interventions for patients with head and neck cancer. Cochrane Database of Systematic Reviews 2013, Issue 7. Art. No.: CD009441. DOI: 10.1002/14651858.CD009441.pub2.

Copyright (C) 2013 The Cochrane Collaboration. Published by John Wiley \& Sons, Ltd.

\begin{abstract}
A B S T R A C T
Background

A diagnosis of head and neck cancer, like many other cancers, can lead to significant psychosocial distress. Patients with head and neck cancer can have very specific needs, due to both the location of their disease and the impact of treatment, which can interfere with basic day-to-day activities such as eating, speaking and breathing. There is a lack of clarity on the effectiveness of the interventions developed to address the psychosocial distress experienced by patients living with head and neck cancer.
\end{abstract}

\section{Objectives}

To assess the effectiveness of psychosocial interventions to improve quality of life and psychosocial well-being for patients with head and neck cancer.

\section{Search methods}

We searched the Cochrane Ear, Nose and Throat Disorders Group Trials Register; the Cochrane Central Register of Controlled Trials (CENTRAL); PubMed; EMBASE; CINAHL; Web of Science; BIOSIS Previews; Cambridge Scientific Abstracts; ICTRP and additional sources for published and unpublished trials. The date of the most recent search was 17 December 2012.

\section{Selection criteria}

We selected randomised controlled trials and quasi-randomised controlled trials of psychosocial interventions for adults with head and neck cancer. For trials to be included the psychosocial intervention had to involve a supportive relationship between a trained helper and individuals diagnosed with head and neck cancer. Outcomes had to be assessed using a validated quality of life or psychological distress measure, or both.

\section{Data collection and analysis}

Two review authors independently selected trials, extracted data and assessed the risk of bias, with mediation from a third author where required. Where possible, we extracted outcome measures for combining in meta-analyses. We compared continuous outcomes using either mean differences (MD) or standardised mean differences (SMD) and 95\% confidence intervals (CI), with a random-effects model. We conducted meta-analyses for the primary outcome measure of quality of life and secondary outcome measures of psychological

Psychosocial interventions for patients with head and neck cancer (Review)

Copyright () 2013 The Cochrane Collaboration. Published by John Wiley \& Sons, Ltd. 
distress, including anxiety and depression. We subjected the remaining outcome measures (self esteem, coping, adjustment to cancer, body image) to a narrative synthesis, due to the limited number of studies evaluating these specific outcomes and the wide divergence of assessment tools used.

\section{Main results}

Seven trials, totaling 542 participants, met the eligibility criteria. Studies varied widely on risk of bias, interventions used and outcome measures reported. From these studies, there was no evidence to suggest that psychosocial intervention promotes global quality of life for patients with head and neck cancer at end of intervention (MD 1.23, 95\% CI -5.82 to 8.27) as measured by the European Organisation for Research and Treatment of Cancer Quality of Life Questionnaire (EORTC QLQ-C30). This quality of life tool includes five functional scales, namely cognitive, physical, emotional, social and role. There was no evidence to demonstrate that psychosocial intervention provides an immediate or medium-term improvement on any of these five functional scales. From the data available, there was no significant change in levels of anxiety (SMD - $0.09,95 \% \mathrm{CI}-0.40$ to 0.23 ) or depression following intervention (SMD -0.03, 95\% CI - 0.24 to 0.19). At present, there is insufficient evidence to refute or support the effectiveness of psychosocial intervention for patients with head and neck cancer.

\section{Authors' conclusions}

The evidence for psychosocial intervention is limited by the small number of studies, methodological shortcomings such as lack of power, difficulties with comparability between types of interventions and a wide divergence in outcome measures used. Future research should be targeted at patients who screen positive for distress and use validated outcome measures, such as the EORTC scale, as a measure of quality of life. These studies should implement interventions that are theoretically derived. Other shortcomings should be addressed in future studies, including using power calculations that may encourage multi-centred collaboration to ensure adequate sample sizes are recruited.

\section{PLAIN LANGUAGE SUMMARY}

\section{Psychosocial interventions for patients with head and neck cancer}

There is a steady rise in the number of people being diagnosed with head and neck cancer. It is more common in men over 60 , but the incidence rate is rising, especially in younger adults (men and women under 40). Survival rates for some cancers in the head and neck area are over $50 \%$. As a result, the quality of life of head and neck cancer patients and how they adjust to life after treatment are becoming increasingly important. Unfortunately life can change greatly for many people following treatment of head and neck cancer due to the obvious change in their appearance, or changes in how they speak and eat. Also, this patient group is known to have high rates of smoking and alcohol use. This combination of more people living with and surviving head and neck cancer, and the high degree of cancer-related issues, has led healthcare professionals to develop programmes to support patients with some of the problems they may experience after treatment. The focus of these programmes is often on addressing emotional or social problems related to the patient's cancer and they are known as 'psychosocial interventions'. This review examines the evidence for the effectiveness of psychosocial interventions to improve quality of life or psychosocial well-being.

We identified seven studies, with a total of 542 adult patients who had head and neck cancer. However, many of the studies had shortcomings in their design or reporting. This has made it difficult to draw reliable conclusions.

Overall, this review did not find any improvement in general quality of life or in levels of anxiety and depression following psychosocial intervention.

In conclusion, there was limited good-quality evidence in this area, making it difficult to draw conclusions about the effectiveness of psychosocial interventions. Future good-quality research is required in this field and should target those in need of psychosocial intervention, in order to guide service development. 


\section{B A C K G R O U N D}

\section{Description of the condition}

Head and neck cancer describes a range of tumours that arise in the head and neck region, which includes the oral cavity, pharynx, larynx, nasal cavity, paranasal sinuses and salivary glands. The worldwide incidence of head and neck cancer exceeds half a million cases annually, ranking it as the fifth most common cancer worldwide (Harrison 2009). With ongoing developments in cancer diagnosis and treatment, the overall survival rate for head and neck cancer has improved marginally but mainly for those from higher socioeconomic backgrounds (CRUK 2011). Head and neck cancer is strongly associated with certain lifestyle factors, mainly tobacco smoking and alcohol consumption (Duffy 2007).

Cancer has a significant impact on the lives of people and is lifedisrupting on many levels. A diagnosis of head and neck cancer, like any other cancer, brings with it significant psychosocial effects including uncertainty and fear. Patients with head and neck cancer have also very specific needs, due to the location of their disease. Many patients face the reality of altered appearance, changes to their speech, swallowing and breathing, and high levels of symptomatology (pain, xerostomia, shoulder dysfunction). The burden of head and neck cancer can manifest in psychosocial distress and social isolation, which can have a negative impact on quality of life (Semple 2008). The prevalence of psychological distress amongst head and neck cancer patients is difficult to estimate, with reported incidences of psychological morbidity varying from $12 \%$ to $45 \%$, depending on the criteria and diagnostic tool utilised (Frampton 2001; Haman 2008; Veer 2010).

As treatments for head and neck cancer develop, modest improvement in survival rates is noted. This presents clinicians with a growing challenge to devise and test interventions that can help people manage the side effects of treatment and the psychosocial impact on their lives.

\section{Description of the intervention}

Over the past three decades a variety of psychosocial interventions have been developed to help head and neck cancer patients cope with the emotional and social impact of the condition. Psychosocial intervention refers to any attempt to provide solutions to the challenges individuals may encounter to their psychological wellbeing when interacting with any element of the social environment (Bessell 2009). Holland 1982 described the goals of these interventions as being to decrease feelings of alienation, lessen feelings of isolation, helplessness and hopelessness, and to assist in clarifying misperceptions and misinformation. These interventions may also promote self management by equipping and empowering patients to take more responsibility for getting well. These interventions vary considerably in their theoretical premise, complexity, content and mode of delivery.

\section{How the intervention might work}

Fawzy 1995 reported on four main types of psychosocial interventions which include: psychoeducational, psychotherapy (individual), cognitive behavioural training and group interventions.

- Psychoeducational interventions vary in the amount of psychosocial information or coping instruction and behavioural training they provide, but the emphasis is generally on educating patients on the disease process and how to cope with the disease.

- Psychotherapy is generally within a one-to-one interaction between a therapist and patient. The aim of psychotherapy is to help the patient reduce distress, gain a sense of mastery over the situation and help in overcoming the practical problems.

- Cognitive behavioural therapy involves identifying and correcting negative thoughts, feelings and behaviours that contribute to psychosocial difficulties. Behavioural and cognitive techniques are employed and are aimed at improving emotional and functional adjustment.

- Group interventions centre on two types of support groups which are either led by a professional or by patients themselves, often referred to as peer support groups. Group interventions are thought to influence self esteem by normalising the experience and reduce feelings of isolation or abnormality, promote adjustment and add meaning to life through helping others and instillation of hope by social comparisons.

\section{Why it is important to do this review}

It is currently recommended in UK government policy that all patients with a cancer diagnosis should "undergo systematic psychological assessment at key points and have access to appropriate psychological support" (NICE 2004, page 13). The National Cancer Comprehensive Network (NCCN) in the USA advocates that all cancer patients should be screened for psychological distress, with the premise that a number may benefit from further psychosocial interventions (Holland 2005). Despite this flourishing body of literature identifying the importance of assessment and intervention, there is a lack of evidence from systematic reviews regarding the effectiveness of psychosocial interventions and which type of interventions are most effective for different groups of patients. A previous integrative review of the head and neck cancer literature tentatively suggested that psychosocial interventions improved quality of life, had a positive impact on emotional adjustment and improved coping skills, but many of these studies had methodological flaws (Semple 2004). In recent years there has been an increase in the number of studies that have developed and tested interventions to improve quality of life, reduce distress and enhance adjustment for patients with head and neck cancer; there- 
fore it is reasonable to suggest that a higher standard of methodology and reporting should be evident in this systematic review. This review will systematically assess the best available evidence on the effectiveness of psychosocial interventions for patients with head and neck cancer. This will provide clinicians with reliable information on the most appropriate interventions, thus promoting adjustment and quality of life for a patient population that has a considerable disease burden.

\section{O B J E C T I V E S}

To assess the effectiveness of psychosocial interventions for adults with head and neck cancer.

\section{METHODS}

\section{Criteria for considering studies for this review}

\section{Types of studies}

Randomised controlled trials (RCTs) and quasi-RCTs of psychosocial interventions for patients with a head and neck cancer diagnosis. 'Quasi-RCT' refers to when the method of allocation is known but not considered as strictly random, such as alternation, date of birth or medical record number.

\section{Types of participants}

Adults (aged 18 or older) with any type or stage of head and neck cancer. There was no restriction on gender, ethnicity, treatment modality, type of setting or stage in the cancer journey.

\section{Types of interventions}

Psychosocial interventions included: psychoeducational, psychotherapy (individual), cognitive behavioural training, supportive and group interventions. For individual or group interventions to be included, they must have comprised of a face-to-face component; but may have been supplemented by written, telephone or computer-assisted learning (demonstrating completion of intervention), at home or in a healthcare setting (or a combination of these) by lay or trained personnel (or a combination of both). Interventions that were solely based on the distribution of informational material (without input from trained or lay personnel) and those that were physical in nature, such as exercise or massage alone, were excluded from this review. Control groups included those who had not received the psychosocial intervention being tested or who had received 'standard care'.

\section{Types of outcome measures}

\section{Primary outcomes}

Quality of life, as measured by an established and/or validated tool, for example, the European Organisation for Research and Treatment of Cancer (EORTC) QLQ-C30.

\section{Secondary outcomes}

The secondary outcome measures included various facets of psychosocial functioning to include the following:

- Anxiety

- Depression

- Coping

- Social functioning

- Self esteem

- Self efficacy

- Body image

- Locus of control

- Adjustment/adaptation

- Life satisfaction

- Satisfaction with the intervention

- Fear of recurrence

All primary and secondary outcome measures must be assessed using an established and/or validated scale, for example: University of Washington Quality of Life (UWQOL), General Health Questionnaire (GHQ), Hospital Anxiety and Depression Scale (HADS), Rotterdam Symptom Checklist, Mental Adjustment to Cancer Scale, Impact of Events Scale and Derriford Appearance Scale.

\section{Search methods for identification of studies}

We conducted systematic searches for randomised controlled trials and quasi-RCTs. There were no language, publication year or publication status restrictions. The date of the search was $17 \mathrm{De}-$ cember 2012.

\section{Electronic searches}

We searched the following databases from their inception for published, unpublished and ongoing trials: the Cochrane Ear, Nose and Throat (ENT) Disorders Group Trials Register; the Cochrane Central Register of Controlled Trials (CENTRAL, The Cochrane Library 2012, Issue 12); PubMed; EMBASE; CINAHL; PsycINFO; LILACS; KoreaMed; IndMed; PakMediNet; CAB Abstracts; Web of Science; BIOSIS Previews; ClinicalTrials.gov; ICTRP; National Cancer Institute Trials database; Cancer Research UK trials database and Google.

We modelled subject strategies for databases on the search strategy designed for CENTRAL. Where appropriate, we combined 
subject strategies with adaptations of the highly sensitive search strategy designed by The Cochrane Collaboration for identifying randomised controlled trials and controlled clinical trials (as described in theCochrane Handbook for Systematic Reviews of Interventions Version 5.1.0, Box 6.4.b. (Handbook 2011)). Search strategies for major databases including CENTRAL are provided in Appendix 1.

\section{Searching other resources}

We scanned the reference lists of identified publications for additional trials and contacted trial authors where necessary. In addition, we searched PubMed, TRIP database and Google to retrieve existing systematic reviews relevant to this systematic review, so that we could scan their reference lists for additional trials. We searched for conference abstracts using the Cochrane Ear, Nose and Throat Disorders Group Trials Register.

\section{Research in progress}

We searched the following online registers of ongoing trials:

- http://controlled-trials.com

- http://clinicaltrials.gov

- http://www.who.int/ictrp

\section{Language}

No language restrictions were placed on trials (unless accurate translation proved problematic). Initially foreign language abstracts were translated and measured against the inclusion and exclusion criteria. Where necessary, methods, results and discussion sections were translated for inclusion in the review, thus enabling completion of the data extraction form.

\section{Data collection and analysis}

\section{Selection of studies}

After removing duplicates, we gave all titles and abstracts of selected studies, retrieved through the electronic searches, an identification code. Pairs of review authors (CS, KP, AN, EMcC, MM) examined the remaining abstracts and independently screened for suitability, according to the following criteria:

- RCT or quasi-RCT;

- psychosocial intervention (based on definition in

Background section and confirmed by CS, AN or both);

- adult (aged 18+) with head and neck cancer; and

- outcome to include a validated measure of quality of life or psychosocial functioning.

Grey literature such as dissertations, theses and conference and poster abstracts were excluded. When it was unclear whether the trial clearly meet the above inclusion criteria, we obtained the full text of the article and checked it for eligibility. When discrepancies arose regarding the inclusion/exclusion of trials between two review authors this was resolved by discussion or, when necessary, by appeal to a third review author. A list of excluded studies and reason for exclusion is included in Characteristics of excluded studies.

\section{Data extraction and management}

Two review authors independently extracted data from the original reports onto pre-designed data extraction forms. Data extraction forms were completed by translators from the Cochrane ENT Disorder Group for one German study and three Chinese studies. Extracted data included the following.

- GENERAL: title of paper, author, year of publication, title, source, country and language of publication, type of report.

- PARTICIPANT: age, gender, ethnicity, cancer stage, location of tumour, treatment modality, sample size and distribution in each arm of the trial, inclusion and exclusion criteria.

- INTERVENTION: description of intervention, mode of delivery, setting, length and frequency of intervention, healthcare professional delivering the intervention, duration of follow-up, control intervention characteristics.

- METHODOLOGICAL QUALITY: see below.

- OUTCOMES: names of the validated tools used and results pertaining to the outcome measures.

\section{Assessment of risk of bias in included studies}

The review authors worked in pairs to assess the methodological quality of the studies selected and, using a quality appraisal checklist, coded them as follows:

\section{Random allocation (selection bias)}

- Low risk, e.g. computer-generated random sequence or table of random numbers

- High risk, e.g. hospital ID, date of birth or surname

- Unclear, e.g. insufficient information about the sequence generation process

\section{Allocation concealment (selection bias)}

- Low risk, e.g. allocation concealment could not be foretold

- High risk, e.g. researcher/healthcare provider aware of the assignment arm of the participant

- Unclear, e.g. insufficient information to permit judgement of 'low' or 'high' 


\section{Blinding (detection bias)}

Blinding of participants to this type of intervention is extremely challenging and in most cases impossible. Nevertheless the outcome assessors can be blinded and this will be coded as:

- Low risk, e.g. blinding ensured and unlikely to be broken

- High risk, e.g. no blinding of outcome assessors or could have been broken

- Unclear, e.g. insufficient information to permit judgement

\section{Incomplete data (attrition bias)}

- Low risk, e.g. no missing outcome data or attrition rate/loss to follow-up clearly accounted for

- High risk, e.g. imbalance of numbers or reasons for missing data not provided

- Unclear, e.g. insufficient information to permit judgement of 'high' or 'low'

\section{Selective reporting}

- Low risk, e.g. primary and secondary outcome measures have been reported in a pre-specified way

- High risk, e.g. not all of the study's pre-defined outcome measures have been reported or report fails to include results from a key outcome that would be expected

- Unclear, e.g. insufficient information to permit judgement of 'low risk' or 'high risk'

\section{Other sources of bias}

- Low risk, e.g. free of other sources of bias

- High risk, e.g. potential sources of bias related to the specific study design

- Unclear, e.g. insufficient information to assess whether an important risk exists

When there was insufficient information we made attempts to contact the study authors for clarification.

We used the information collected in the 'Risk of bias' tool available in RevMan 5 (RevMan 2012) to assess the quality of each study.

\section{Measures of treatment effect}

We entered data into RevMan 5. All the reported data in the included studies were continuous. We extracted means and standard deviations (SD) and, where appropriate, converted standard errors (SE) into standard deviations for meta-analysis using $95 \%$ confidence intervals and a random-effects model. When different scales were used to measure the same outcome, we used the standardised mean difference (SMD) instead of the mean difference (MD).

\section{Dealing with missing data}

When missing data or study discrepancies were evident, we made attempts (where possible) to contact the original investigators for further information.

\section{Assessment of heterogeneity}

Studies in this review were derived from a heterogeneous group of psychosocial interventions which differed in their theoretical premises. However, when the essential characteristic of the intervention was based on psychosocial elements to improve well-being, this rendered it appropriate to combine data assuming they were assessing the same outcome. We assessed heterogeneity between studies using the $\mathrm{I}^{2}$ statistic. We considered an $\mathrm{I}^{2}$ value greater than $50 \%$ as indicative of heterogeneity. Where marked heterogeneity was found, we investigated sources. In the event of substantial clinical, methodological or statistical heterogeneity, we did not combine study results by means of meta-analysis. We used random-effects models for all meta-analyses.

\section{Assessment of reporting biases}

We did not generate a funnel plot because there were too few studies in the meta-analysis carried out in this review.

\section{Data synthesis}

Some outcomes were measured by a range of scales, e.g. depression. We first conducted a meta-analysis of data from the same scale, followed by meta-analyses combining data from different scales. We used SMD with a random-effects model to counteract heterogeneity in these studies. Where there were insufficient data reported, we carried out qualitative synthesis.

\section{Subgroup analysis and investigation of heterogeneity}

It was our intention to conduct the following subgroup analyses where data were available.

- Stage in cancer journey (potentially curative, palliative)

- Healthcare professional delivering intervention (nurse, psychologist, counsellor, allied health professional)

- Method of delivery (individual, group, telephone)

- Duration: short-term (i.e. less than three months), medium-term (i.e. three to six months) or long-term (more than six months)

- Separate analysis on studies by type as described in the Background section (Fawzy 1995)

When the data had been extracted it was clear that insufficient data were available to conduct these subgroup analyses. 


\section{Sensitivity analysis}

When higher-risk studies were identified by the 'Risk of bias' tool we conducted a sensitivity analysis, including only those studies with suspected low risk of bias. If a study was initially determined as having an 'unclear' risk of bias due to lack of information on what happened in the study, then we sought further clarity from the authors.

\section{RES U L T S}

\section{Description of studies}

See: Characteristics of included studies; Characteristics of excluded studies.

\section{Results of the search}

Figure 1 illustrates the process of study selection for the review and was lasted updated on 17 December 2012. From the electronic searches we retrieved 1035 references, although this number dropped to 851 references after duplicates were removed. After an initial subject/title screen 241 references remained. Two members of the review team independently examined the abstracts of the remaining 241 papers to remove obviously irrelevant studies that were: 
Figure I. Process of sifting search results and selecting studies for inclusion.

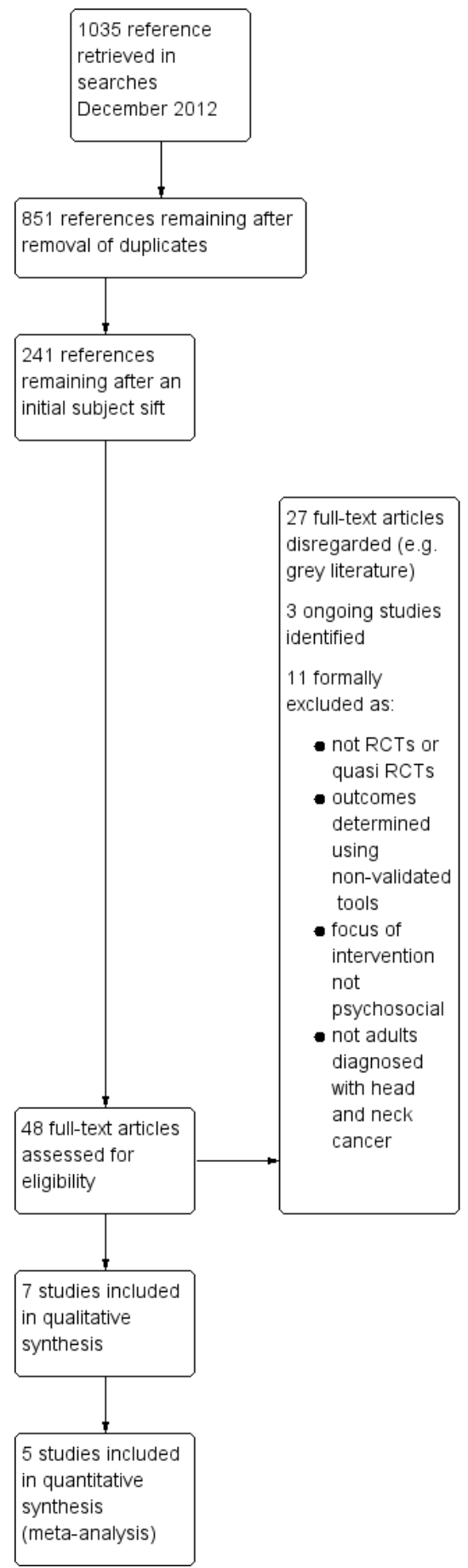

Psychosocial interventions for patients with head and neck cancer (Review)

Copyright $\odot 2013$ The Cochrane Collaboration. Published by John Wiley \& Sons, Ltd. 
- guidelines or a discussion paper and NOT a research paper;

- reviews or meta-analyses;

- in children and not adults;

- in non-cancer patients;

- prevention or screening papers.

Following this, we scrutinised 48 full papers and disregarded a further 27 (e.g. grey literature). Three studies were identified as ongoing (see Characteristics of ongoing studies). We formally excluded 11 studies for one of the following reasons:

- not RCTs or quasi-RCTs;

- outcomes determined using non-validated tools;

- focus of the intervention was not psychosocial;

- participants were not adults diagnosed with head and neck cancer.

Seven studies were deemed suitable for inclusion.

\section{Included studies}

Of the seven studies that were included, two were conducted in Canada (Allison 2012; Katz 2004), three in China (Bai 2004; Fan 2006; Yongqin 2009), one in the USA (Duffy 2006) and one in the UK (Humphris 2012). Four of these studies were written in English (Allison 2012; Duffy 2006; Humphris 2012; Katz 2004) and three in Chinese (Bai 2004; Fan 2006; Yongqin 2009). Data extraction forms for the three Chinese papers were completed by a Chinese author for the Cochrane Ear, Nose and Throat Disorder Group.

\section{Design}

All of the included studies in the review were RCTs, therefore all seven reported baseline assessment and a post-intervention assessment for the experimental and control groups (Allison 2012; Bai 2004; Duffy 2006; Fan 2006; Humphris 2012; Katz 2004; Yongqin 2009). All studies had one post-intervention assessment point, with the exception of Humphris 2012 and Katz 2004 which had two. All of the studies had one experimental arm.

\section{Participants}

The included studies recruited between 19 (Katz 2004) and 184 (Duffy 2006) participants. A total of 542 participants were recruited from the seven included studies. The mean number of participants was 77. Fan 2006 did not report on gender, therefore from the remaining six studies there were 364 male and $143 \mathrm{fe-}$ male participants. An overview of each study can be found in the Characteristics of included studies table.

\section{Disease characteristics}

All studies excluded participants who were in a terminal phase of their illness/distant metastasis. Some of the studies included participants with a range of tumour sites within the head and neck region ( $\mathrm{n}=245$ ) (Allison 2012; Duffy 2006), whereas participants in Bai 2004 and Fan 2006 had solely nasopharyngeal cancer (n = 117). Participants in Humphris 2012 and Katz 2004 had a diagnosis of oral cancer $(n=96)$ and the Yongqin 2009 cohort had laryngeal or hypopharyngeal carcinoma $(n=84)$.

\section{Psychological screening and inclusion/exclusion criteria}

Two of the studies (Allison 2012; Duffy 2006) screened for psychological distress prior to accrual. Allison 2012 defined psychological distress as a score of more than seven on the anxiety and/ or depression scale of the Hospital Anxiety and Depression Scale (HADS) (Zigmond 1983), with the aim of targeting interventions for those with pre-existing levels of distress. Duffy 2006 included participants who screened positive for one or more of three health problems: smoking, alcohol or depression. Study eligibility for probable depression was defined as a score of four or more on the Geriatric Depression Scale-Short Form (Sheikh 1986).

Four trials excluded participants with a history of psychological problems (Allison 2012; Duffy 2006; Fan 2006; Humphris 2012). One specifically excluded those who had previously been diagnosed with depression and were currently undergoing any antidepressive therapy (Allison 2012). Both Duffy 2006 and Humphris 2012 excluded participants with unstable psychiatric/mental conditions such as suicidal ideation/acute psychosis, whereas Fan 2006 excluded any participants with a history of mental illness.

\section{Intervention}

A number of different categorisations of psychosocial intervention are used to enhance adjustment to cancer. As noted earlier, Fawzy 1995 reported on four main types of psychosocial intervention, which include: psychoeducational, psychotherapy (individual), cognitive behavioural training and group interventions. Nonetheless, in practice not all the psychosocial interventions fit neatly into these different categories. For example, psychoeducation is often a component in cognitive behavioural interventions and a cognitive behavioural intervention could be delivered in a group format. Despite this, the studies within a systematic review need to be considered homogenous to ensure that a combined analysis of pooled estimates is valid. Therefore, this review is founded on the principle that a psychosocial intervention is a function of a supportive relationship between a 'trained helper' and an individual diagnosed with cancer, using some specific technique(s) that has a potential to alter or change psychological constructs. 
The supportive interaction between a 'trained helper' and an individual participant is evident in all included studies, which had the common aim of improving quality of life and decreasing psychosocial distress following a diagnosis of head and neck cancer. None of the included studies used group, family or couple-based therapy. However, the theoretical bases within the interventions varied considerably. Two studies referred to the intervention as psychoeducation (Allison 2012; Katz 2004). Five studies described utilising CBT or recognised different aspects of the CBT model to form part of the psychosocial intervention (Bai 2004; Duffy 2006; Fan 2006; Humphris 2012; Yongqin 2009). Three studies stated that they combined physical exercise with the psychosocial intervention (Bai 2004; Fan 2006; Yongqin 2009) to promote psychological well-being, whilst Duffy 2006 included pharmacological intervention if required.

Furthermore, the Katz 2004 study took the form of a brief, preparatory intervention timed immediately before surgery for oral cancer, with a further supportive session on postoperative day two or three. The other studies were designed to address psychosocial distress experienced by people who had completed treatment for head and neck cancer (Allison 2012; Bai 2004; Duffy 2006; Fan 2006; Humphris 2012; Yongqin 2009).

\section{Frequency and mode of intervention delivery}

There was considerable heterogeneity regarding duration and mode of intervention delivery in these studies. Nonetheless, all of the studies had a face-to-face component of the intervention delivered by a professional. Two studies (Duffy 2006; Katz 2004) had one face-to-face contact, supplemented with telephone contacts. Duffy 2006 had the initial assessment conducted face-to-face, followed by 9 to 11 telephone sessions, whereas the first intervention session in Katz 2004 was via telephone and the second session was delivered face-to-face. These two studies supplemented their intervention with printed material. Katz 2004 produced a psychoeducational booklet ( 95 pages) and Duffy 2006 had a detailed workbook with CBT exercises. Allison 2012 also provided a manual, plus audio material and music for relaxation.

Interventions were delivered by a variety of professionals. The majority of studies referred to trained therapists delivering the intervention (Allison 2012; Duffy 2006; Fan 2006; Humphris 2012). In three studies it was a nurse who delivered the intervention (Duffy 2006; Humphris 2012; Katz 2004). In Fan 2006, a multidisciplinary team delivered the intervention. Three studies did not provide information on the discipline of the professionals involved (Allison 2012; Bai 2004; Yongqin 2009).

\section{Control groups}

In all seven studies participants had been randomised to either the intervention or control group. Nonetheless, the control groups differed across the studies, as five studies had standard usual care as the control (Bai 2004; Fan 2006; Humphris 2012; Katz 2004; Yongqin 2009). In the Allison 2012 study, the control group had an attention placebo (i.e. received a similar number of contacts and were allowed to discuss cancer-related issues) and Duffy 2006 used enhanced usual care to include a brief assessment and counselling session with onward referrals as necessary for supportive/ psychiatric services as their control group.

\section{Outcomes}

A wide variety of outcomes measures were used in the seven studies, measured at different time points and they also varied in the length of follow-up time. Nevertheless, each study either included a quality of life measure (Bai 2004; Fan 2006; Humphris 2012; Katz 2004; Yongqin 2009) and/or psychological distress measure (Allison 2012; Duffy 2006; Humphris 2012; Katz 2004). Quality of life was measured in five out of the seven included studies. Three studies (Bai 2004; Humphris 2012; Katz 2004) used the extensively validated European Organisation for Research and Treatment of Cancer (EORTC) QLQ-C30 (Aaronson 1993) to measure health-related quality of life. The EORTC QLQ-C30 is a widely used and validated health-related quality of life measure consisting of five functional scales (physical, role, cognitive, emotional and social), a separate quality of life domain and three symptom scales measuring fatigue, pain and emesis. Fan 2006 modified the EORTC QLQ-C30 and Yongqin 2009 used a quality of life tool for Washington medical college students. Therefore, we did not extract from either of these two studies for the meta-analysis. The three studies that used the EORTC QLQ-C30 all had a relatively short-term post-intervention measuring point of either three or four months (Bai 2004; Humphris 2012; Katz 2004). Four studies measured depression (Allison 2012; Duffy 2006; Humphris 2012; Katz 2004) but there was variation in the tools used. Two studies (Allison 2012; Humphris 2012) used the Hospital Anxiety and Depression Scale (HADS) (Zigmond 1983), whilst Duffy 2006 used the Geriatric Depression Scale-Short Form (Sheikh 1986) and Katz 2004 used Centre for Epidemiologic Studies Depression Scale (CES-D) (Radloff 1977). The medium-term post-intervention measurement point for all four studies was between three and six months. Similarly, three of these studies measured anxiety between three and four months post-intervention, with Allison 2012 and Humphris 2012 using the anxiety subscale of the HADS, and Katz 2004 using the State-Trait Anxiety Inventory (Spielberger 1980). Three studies provided appropriate data for meta-analyses of quality of life (Bai 2004; Humphris 2012; Katz 2004), whereas four provided data suitable for meta-analyses of depression (Allison 2012; Duffy 2006; Humphris 2012; Katz 2004), with three providing data for meta-analyses of anxiety (Allison 2012; Humphris 2012; Katz 2004).

\section{Excluded studies}


We formally excluded 11 studies after two review authors (CS, MM) assessed the full texts (see Characteristics of excluded studies). Reasons for excluding these studies were as follows:

- not a psychosocial intervention, e.g. educational material alone, massage;

- not a RCT or quasi-RCT;

- mixed cancer sample but no separate data for head and neck cancer participants;

- did not use a validated measure relevant to this review.

\section{Risk of bias in included studies}

We assessed all studies for risk of bias using the RevMan 5 'Risk of bias' tool (RevMan 2012). We rated only one study included in this review (Allison 2012) as being at an entirely low risk of bias. The lack of important details for a number of the criteria, such as method of random sequence generation and allocation, in the remaining studies (Bai 2004; Duffy 2006; Fan 2006; Humphris 2012; Katz 2004; Yongqin 2009) precluded an adequate assessment of their quality and the possibility of bias exists. We viewed three studies as having high risk of bias for some aspects of the assessment criteria (Bai 2004; Duffy 2006; Katz 2004). Details of the 'Risk of bias' assessment for the seven included studies are outlined in the Characteristics of included studies and summarised in Figure 2 and Figure 3.

Figure 2. 'Risk of bias' graph: review authors' judgements about each risk of bias item presented as percentages across all included studies.

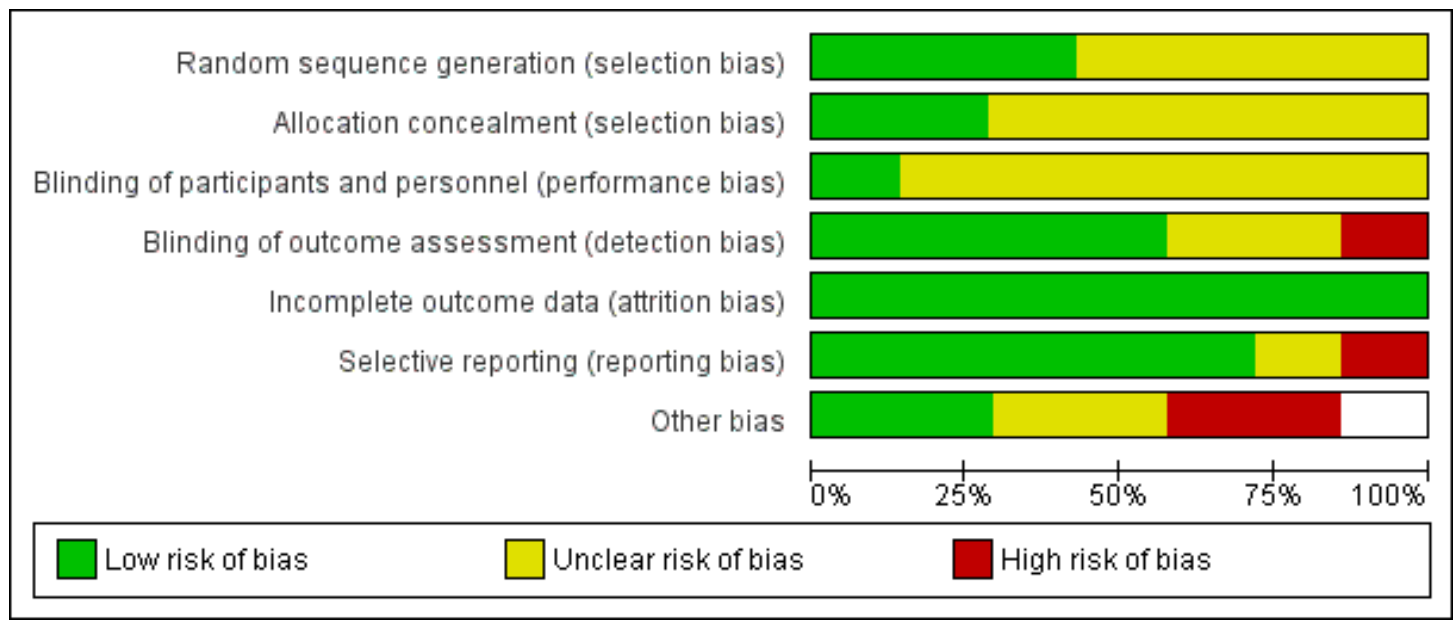


Figure 3. 'Risk of bias' summary: review authors' judgements about each risk of bias item for each included study.

\begin{tabular}{|c|c|c|c|c|c|c|c|}
\hline & 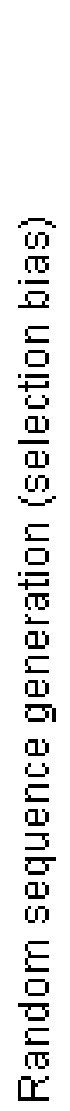 & 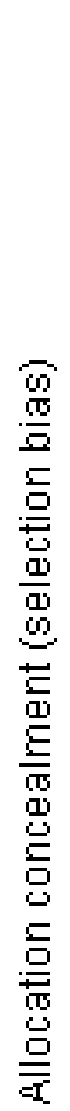 & 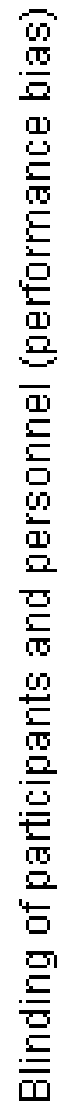 & 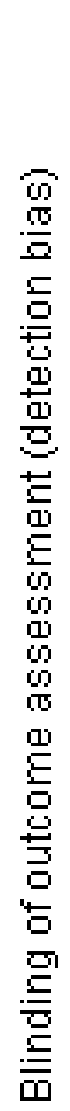 & 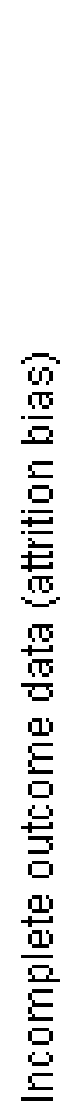 & 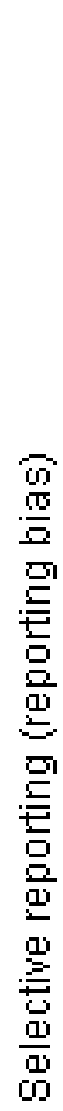 & 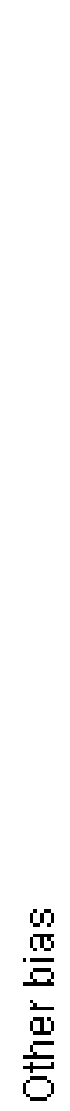 \\
\hline \multicolumn{8}{|l|}{ Allison 2012} \\
\hline Bai 2004 & $?$ & $?$ & $?$ & & & & \\
\hline Duffy 2006 & $?$ & $?$ & $?$ & $?$ & & & \\
\hline Fan 2006 & $?$ & $?$ & $?$ & & & & $?$ \\
\hline Humphris 2012 & + & + & $?$ & & $A$ & $?$ & $?$ \\
\hline Katz 2004 & + & $?$ & $?$ & & & & \\
\hline Yongqin 2009 & $?$ & $?$ & $?$ & $?$ & + & + & \\
\hline
\end{tabular}

Psychosocial interventions for patients with head and neck cancer (Review)

Copyright $\odot 2013$ The Cochrane Collaboration. Published by John Wiley \& Sons, Ltd. 


\section{Allocation}

The inclusion criterion was that all studies should be randomised. All seven included studies stated that randomisation had occurred. Nonetheless, only three studies clearly stipulated that a method of random sequence generation had taken place (Allison 2012; Humphris 2012; Katz 2004). Allison 2012 and Humphris 2012 used a computer-generated randomisation list and Katz 2004 used a random number table and we therefore classified them as low risk of bias against this criteria. We classified the other studies as 'unclear', having not clarified the method used.

With regard to allocation concealment, two studies clearly stated a method that we considered to be at low risk of bias (Allison 2012; Humphris 2012). The generation of allocation sequence for both of these studies was performed by an individual who was independent of the project delivery (e.g. independently prepared by the faculty statistician). The remaining studies did not provide sufficient information on the method of concealment and we have reported them as at 'unclear' risk of bias.

\section{Blinding}

Due to the nature of the intervention being delivered (i.e. psychosocial care) it is difficult to blind the participant and personnel to the allocated intervention. Allison 2012 was the only study which kept the participants blind to whether they were receiving the experimental or control intervention, being an attention placebo.

The blinding of assessors (data collectors) was reported in four studies, which we considered as at low risk bias (Allison 2012; Fan 2006; Humphris 2012; Katz 2004), however it was not mentioned by Duffy 2006 and Yongqin 2009. We felt the Bai 2004 study to have a high risk of bias for blinding of assessors as the researchers and personnel worked collaboratively to accomplish outcome assessment.

\section{Incomplete outcome data}

A high level of missing data or poor handling of missing data leads to attrition bias. Fortunately for this review, when there was loss to follow-up, all studies clearly recorded the number of participants lost, with reasons provided (Allison 2012; Duffy 2006; Humphris 2012; Katz 2004). This demonstrated that attrition was balanced across groups for these four studies. No loss of participants to follow-up was reported in the three Chinese studies (Bai 2004; Fan 2006; Yongqin 2009).

\section{Selective reporting}

There was no evidence of selective reporting in five of the seven studies, with all outcome data reported (Allison 2012; Bai 2004;
Fan 2006; Katz 2004; Yongqin 2009). Duffy 2006 reported the results of two scales (AUDIT and the Geriatric Depression ScaleShort Form) as dichotomous data but through personal communication continuous data were provided for the depression outcome measure (CES-D) to enable inclusion in the meta-analysis. Humphris 2012 provided only a narrative summary of health-related quality of life data (EORTC QLQ-C30) but again through personal communication continuous data were provided for metaanalysis.

\section{Other potential sources of bias}

We assessed two studies (Duffy 2006; Katz 2004) as having additional risk of bias. Duffy 2006 received funding from a pharmaceutical company and two products which were part of the treatment management protocol for the intervention are produced by this company. Also, Katz 2004 had a small sample size $(\mathrm{n}=19)$ and used a large battery of questionnaires with multiple statistical tests conducted.

\section{Effects of interventions}

The following section provides a description of the meta-analysis for the primary outcome (quality of life) and the secondary outcome (anxiety and depression). A brief narrative of the studies that did not present adequate data for meta-analyses is also presented. Of the studies with sufficient extractable data to be included in the meta-analysis, all had follow-up periods varying between three and six months and we considered this as medium-term followup (Allison 2012; Bai 2004; Duffy 2006; Humphris 2012; Katz 2004).

\section{Quality of life}

Quality of life was measured in five out of the seven included studies. Three studies (Bai 2004; Humphris 2012; Katz 2004) used the extensively validated European Organisation for Research and Treatment of Cancer Quality of Life Questionnaire (EORTC QLQ-C30) to measure health-related quality of life. Fan 2006 modified the EORTC QLQ-C30 and Yongqin 2009 used a quality of life tool for Washington medical college students. Therefore, we did not extract data from either of these two studies for the meta-analysis.

The EORTC QLQ-C30 is presented with five functional scales (physical, role, cognitive, emotional and social), a separate global quality of life domain and three symptom scales measuring fatigue, pain and emesis. We also conducted meta-analyses for the five functional scales and the global quality of life domain for the three studies with extractable data, in keeping with the objectives of this review (Bai 2004; Humphris 2012; Katz 2004). There was

Psychosocial interventions for patients with head and neck cancer (Review) 
no evidence of any benefit following psychosocial intervention on global quality of life or on any of the five functional scales. A more comprehensive review of the results is reported below under two headings: medium-term follow-up and end of intervention.

\section{Medium-term follow-up (three to six months post- intervention)}

The results of the meta-analysis for the physical function scale of the EORTC QLQ-C30 at medium-term follow-up (three to six months) can be viewed in Figure 4. This combines Bai 2004, Humphris 2012 and Katz 2004, totaling 138 participants, and demonstrates no statistically significant evidence favouring the intervention group (mean difference (MD) 6.28, 95\% confidence interval (CI) -10.05 to 22.60) (Analysis 1.1). This finding must be considered with caution due to the small number of studies, small sample sizes, the large CI and the moderate degree of statis- tical heterogeneity between study estimates ( $\left.\mathrm{P}=0.06, \mathrm{I}^{2}=65 \%\right)$. Also, Bai 2004 has been classified as being at high risk of bias, as details of how the random sequence was generated, allocation and blinding of outcomes were not provided. In addition, Bai 2004 did not provide a sample size calculation, nor was there clarification of whether the target recruitment was reached to identify statistically significant findings. Similarly, for the other four functional scales there is no demonstrable evidence that psychosocial intervention improves emotional functioning (MD 2.54, 95\% CI -4.81 to 9.89) (Figure 5; Analysis 1.2), social functioning (MD $-2.36,95 \%$ CI -11.36 to 6.65 ) (Analysis 1.3), role functioning (MD $-1.43,95 \% \mathrm{CI}-7.31$ to 4.46 ) (Analysis 1.4 ) or cognitive functioning (MD -4.23, 95\% CI -12.48 to 4.03) (Analysis 1.6) at three to six months following psychosocial intervention. The analysis did not provide evidence for the effectiveness of psychosocial intervention to improve global quality of life (MD -2.57, 95\% CI -13.54 to 8.40 ) (Analysis 1.5) at this time juncture.

Figure 4. Forest plot of comparison: I Intervention verus control, outcome: I.3 EORTC QLQ C30 - Physical function. Medium-term follow-up

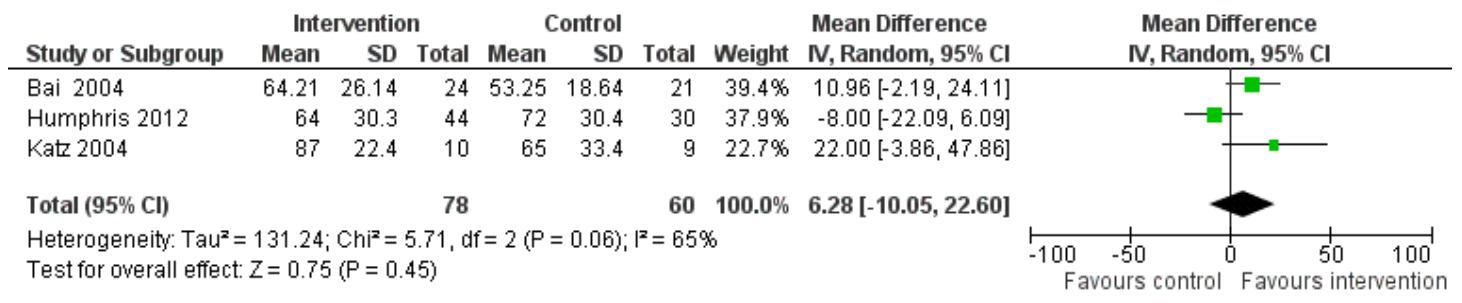

Figure 5. Forest plot of comparison: I Intervention verus control, outcome: I.6 EORTC QLQ C30 Emotional function. Medium-term follow-up.

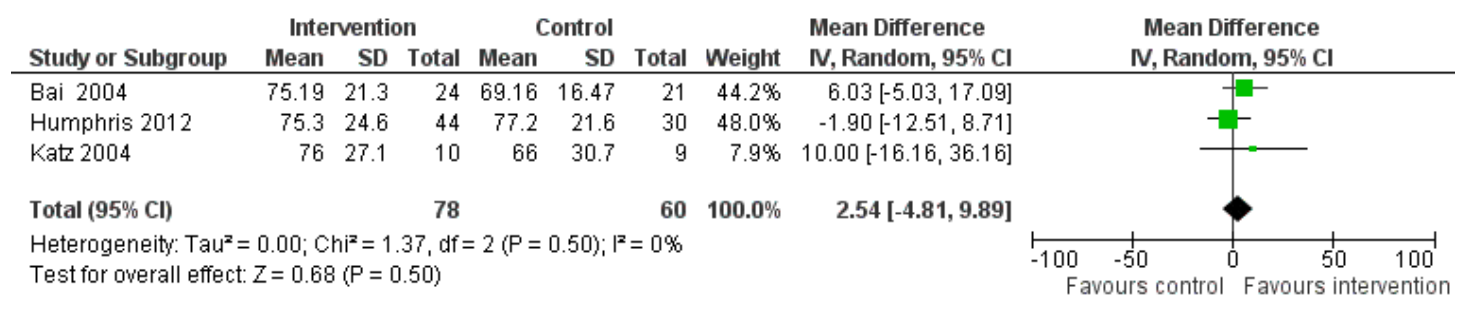

\section{End of intervention}

Humphris 2012 and Katz 2004 did have data collection points immediately at the end of intervention. Using end of intervention data, these findings do show a small trend towards improved cognitive function on the EORTC QLQ-C30 subscale immedi- 
ately following intervention (MD $2.78,95 \% \mathrm{CI}-4.82$ to 10.37 ) (Figure 6; Analysis 1.7) but this did not reach statistical significance. This is an important result, suggesting that when head and neck cancer patients' anxieties and fears are addressed they are able to demonstrate some improvement in their planning ability and re-engage in their normal activities in the period immediately following intervention, although this effect is not maintained a few months later. There was also a small trend towards improvement in physical function at end of intervention (MD 6.74, 95\% CI 2.24 to 15.71 ) (Analysis 1.8) but this was not statistically significant. There was no directional change in any of the other EORTC QLQ-C30 subscales when exploring the effectiveness of the psychosocial programmes at the end of intervention (Analysis 1.9; Analysis 1.10; Analysis 1.11). There was no difference in global quality of life between the two groups at this time point (Analysis 1.12).

\section{Figure 6. Forest plot of comparison: I Intervention verus control, outcome: I.9 EORTC QLQ C30 -}

Cognitive function (end of intervention).

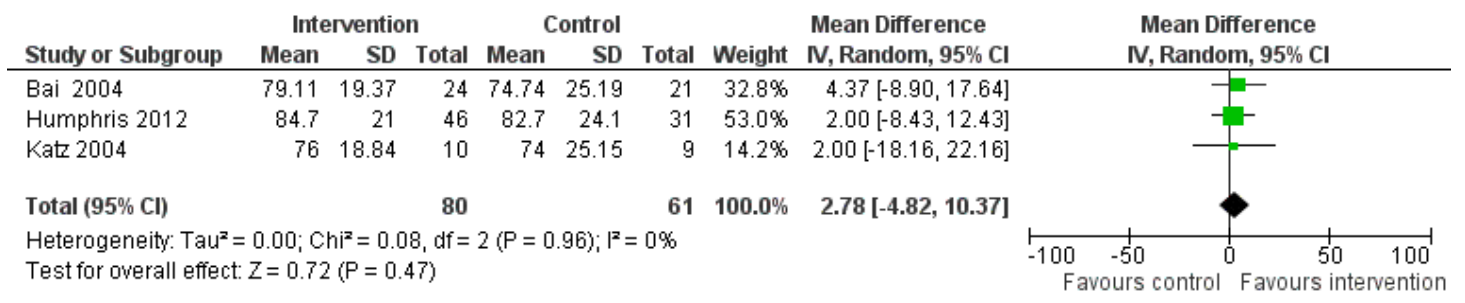

We performed no subgroup analyses according to theoretical basis or type of intervention, method of delivery (e.g. individual versus group), discipline of professional delivering intervention or duration of follow-up (i.e. short, medium, long-term) for the primary outcome measure quality of life. Furthermore, the focus of these interventions all varied. Katz 2004 used the format of a brief, preparatory intervention timed immediately before surgery for oral cancer. Humphris 2012 focused specifically on anxiety and fear of recurrence in patients previously treated with surgery with or without adjuvant therapies for oral and oropharyngeal cancer. Bai 2004 combined cognitive behavioural therapy (CBT) and exercise therapy following treatment with radiotherapy for nasopharyngeal cancer.

\section{Brief narrative synthesis of studies not included in the quality of life meta-analysis}

The two remaining studies that reported on quality of life but without extractable data for meta-analysis were Fan 2006 and Yongqin 2009. Fan 2006 delivered a complex psychosocial intervention, over a two-year duration. This intervention included not only a psychological component based on CBT techniques and social skills training but physical fitness training. This study did show statistically significant improvements in mental state (MD 2.95, 95\% CI 0.91 to $4.99, \mathrm{P}<0.05)$ and self evaluation of quality of life (MD - $0.6,95 \%$ CI -1.22 to $0.16, \mathrm{P}<0.01$ ) for the intervention group. Akin to the other studies in this review, the reliability of this finding remains limited due to the small sample sizes $(n=72)$ but also due to the risk of bias for random sequence generation and allocation concealment.

Yongqin 2009 focused their study on patients who had undergone total laryngectomy, using a CBT approach and physical exercise to promote mastery of managing physical and social situations and promote quality of life over a two-year period. This intervention did demonstrate a positive impact on the intervention group for engaging with activities of daily living (MD 1.22, 95\% CI 0.49 to $1.95, \mathrm{P}=0.001$ ) and recreation (MD $1.5,95 \% \mathrm{CI} 0.88$ to $2.16, \mathrm{P}$ $=0.00001)$ at the end of intervention. The authors have provided limited information on study design, thus the risk of bias remains somewhat unclear for this study.

\section{Anxiety}

Effects on anxiety were measured in three studies (Allison 2012; Humphris 2012; Katz 2004) and data were extractable from these studies for inclusion in a meta-analysis. In Allison 2012 and Humphris 2012 anxiety was measured using the Hospital Anxiety and Depression Scale (HADS), commonly used in cancer populations. These studies both report anxiety scores at three to four months post-intervention (medium-term follow-up). The remaining study (Katz 2004) also used a validated tool but one that is not as widely reported in the cancer literature, namely the StateTrait Anxiety Inventory. This tool has two subscales as its name suggests, one for state anxiety and the other for trait anxiety. The state anxiety score at three months post-intervention has been used 
in this meta-analysis. Analysis 1.13 shows that the overall effect size is not statistically significant (standardised mean difference (SMD) $-0.09,95 \%$ CI -0.40 to 0.23 ). Heterogeneity was low (I $2=0 \%$ ). This finding is replicated at end of intervention (SMD $-0.09,95 \%$ CI -0.41 to 0.23$)$ with low heterogeneity $\left(\mathrm{I}^{2}=0 \%\right)$ (Analysis 1.14).

\section{Depression}

We produced a combined analysis of results across four studies (n $=335$ ) that represent depression using validated measures (Allison 2012; Duffy 2006; Humphris 2012; Katz 2004). Three different tools were used to measure depression (see Included studies, subsection on outcome measures). These four studies all measured depression at three to six months post-intervention (medium-term follow-up). The analysis did not provide evidence of the effectiveness of psychosocial intervention to alleviate depression amongst patients who had treatment for head and neck cancer. The effect size was not significant (SMD -0.00, 95\% CI -0.22 to 0.21 ) at end of intervention (Analysis 1.16) or at medium-term follow-up (SMD -0.03, 95\% CI -0.24 to 0.19) (Analysis 1.15), with low statistical heterogeneity in the outputs of pooled estimates $\left(\mathrm{I}^{2}=\right.$ $0 \%)$.

The four studies that reported on the effectiveness of psychosocial intervention to alleviate distress (anxiety and depression) all had extractable data for medium-term follow-up. Nonetheless, as the specific focus of the intervention varied between the studies, from preparation before and directly after surgery (Katz 2004) to fear of recurrence (Humphris 2012) (as noted above in the quality of life section), this may, in part, have contributed to the inconclusiveness as to the effectiveness of psychosocial intervention for patients with head and neck cancer.

\section{Other secondary outcome measures}

\section{Brief narrative synthesis of studies with additional secondary} outcome measures not suitable for meta analyses

In addition to assessing the effectiveness of their psychoeducational intervention for reducing anxiety and depression (see findings above), in Allison 2012 testing took place for the following two secondary outcomes measures: self esteem and coping. The respective validated tools were used: the Rosenberg Self-Esteem scale (Rosenberg 1965) and Ways of Coping Checklist (Folkman 1980). The Ways of Coping Checklist assesses thoughts and actions used to cope with stressful cancer-related events. It measures coping processes, rather than coping dispositions or styles. The findings demonstrate that both the intervention group and the attention-placebo (control) group had improved self esteem and coping. Therefore, the results suggest that patients receiving the psychosocial intervention therapy had no improvements in self esteem and coping beyond those noted in the placebo group, who had one to three meetings where they could discuss cancer-related issues of their choice with information provided on support services.

The intervention used in the Humphris 2012 study is described in detail in a previous publication (Humphris 2008). The Adjustment to the Fears, Threat and Expectation of Recurrence (AFTER) intervention programme included assessing worry using the threeitem Worry of Cancer Scale (Easterling 1989) and adjustment to cancer using the 40-item Mental Adjustment to Cancer Scale (Watson 1989). Fear of recurrence as assessed using the Worry of Cancer Scale did improve significantly in the intervention group as opposed to the control group at the end of intervention (effect size $=0.5$ ). Similarly, the anxious preoccupation subscale on the Mental Adjustment to Cancer Scale demonstrated a significant difference at the end of intervention (effect size $=0.7$ ), but this was not sustained at four months post-intervention. A small, positive, indirect effect of the intervention was found using a structured equation analysis for the Worry of Cancer Scale at four months follow-up. However, no significant changes were noted on the fighting spirit and hopelessness subscales of the Mental Adjustment to Cancer Scale at immediate or medium-term follow-up in the intervention group relative to those receiving standard care.

In Katz 2004 the focus of the psychoeducational intervention was to facilitate preparation for newly diagnosed oral cancer pending surgical treatment and to promote coping with the consequences of invasive surgery. In this pilot study, totaling 19 participants, Katz 2004 used an assessment package with 11 different tools (see Characteristics of included studies). As well as reporting on quality of life and psychological distress (see above), the following secondary outcome measures were assessed: social functioning, adjustment to cancer, body image, self efficacy and life satisfaction. These pilot data revealed that the intervention group had higher satisfaction with their appearance at three months followup compared to the control group. No evidence of effectiveness was demonstrated on any of the remaining secondary outcome measures. Caution must be used in interpreting these findings, given the small sample size with multiple analyses from the large battery of questionnaires used. Furthermore, there is no mention of a counter-balancing technique being used, which randomises the order of the questionnaires, to control for fatigue effects when using a large number of questionnaires.

\section{DISCUSSION}

\section{Summary of main results}

This review includes seven randomised controlled trials (RCTs), totaling 542 participants, denoting a paucity of research evaluating psychosocial interventions for head and neck cancer patients. From these studies, there is no evidence that psychosocial intervention promotes global quality of life for patients with head and 
neck cancer. The analysis did not provide evidence to support any improvement of physical, role, cognitive, emotional or social functioning by psychosocial intervention. Also, from the limited data available, there were no demonstrable changes in levels of anxiety or depression following intervention for patients with head and neck cancer.

RCTs in this review had notable variations in the focus of the intervention, ranging from preparation for post-surgical changes to dealing with fear of recurrence. Sometimes the intervention described in the seven studies overlapped in the theoretical premise to include aspects of cognitive behavioural therapy and psychoeducation. Heterogeneity was also present when the mode of intervention delivery and assessment tools were considered. Given this high level of variation, we have not conducted further subgroup analysis. In this review we could not determine the optimal duration (number of sessions) or intensity (regularity of sessions) of therapy or who was best placed to facilitate such interventions (e.g. nurse, psychologist). In light of this, it is challenging to present a clear statement about effectiveness, as currently there is a lack of convincing evidence to support universal implementation of psychosocial interventions to improve quality of life or reduce psychosocial distress. In conclusion, much remains to be done before firm evidence on the effects of psychosocial intervention for patients with head and neck cancer is available.

\section{Overall completeness and applicability of evidence}

There is a lack of generalisability of the evidence from this review. This is due to the limited number of studies with small sample sizes, reporting on different categories of psychosocial intervention and using a wide range of outcome measures. As previously noted, there was only sufficient evidence to pool the results for the primary outcome, namely quality of life, and for two secondary outcomes, anxiety and depression. Furthermore, not all the studies had extractable data for these outcomes. For example, the main statistical finding of this review is based on quality of life data, with extractable data from only $26 \%$ (141 out of 542 ) of the participants recruited, totaling three out of the seven included studies. The statistical analysis of depression represented a greater number of the overall participants in this review $(62 \%, 335$ out of 542 participants), whereas the anxiety outcome measure only represents data pooled from $29 \%$ of the overall participants (157 out of 542 participants). There were insufficient data to address the remainder of the secondary outcomes in meta-analyses in this review, such as coping, social functioning, self esteem, self efficacy, body image, locus of control, adjustment/adaptation, life satisfaction and satisfaction with the intervention.

This review has highlighted a wide range of psychosocial interventions, which have been developed to promote quality of life and reduce distress following treatment for head and neck cancer. However, the importance of diversity must be recognised, in that there is a universal understanding that facilitation of posttreatment coping may not be met by 'a one size fits all' approach. Counter-balancing this, the wide divergence in this small number of RCTs with small samples limits conclusive evidence on which type or what aspects of the intervention are most applicable.

There was also considerable disparity in what constituted the control group for each study. Although Bai 2004, Fan 2006, Humphris 2012, Katz 2004 and Yongqin 2009 all report usual care, the model of care can vary considerably between countries. On the other hand, Allison 2012 had an attention placebo as the control group, which allowed participants to discuss cancer-related issues and to be informed of available support services. Similarly, Duffy 2006 used enhanced usual care, to include referral to targeted support services and a handout of relevant resources. Interestingly, neither of these studies (Allison 2012; Duffy 2006) with enhanced usual care demonstrated any significant improvement in anxiety or depression compared to the experimental arm.

A further disparity that must be acknowledged is that Allison 2012 and Duffy 2006 screened for psychosocial distress prior to accrual, whereas this was not undertaken by the other studies. We would advocate a mechanism of screening for a pre-identified need for the targeted intervention. This would not only mirror clinical practice but encourage best utilisation of resources.

Regarding the applicability of the evidence, it is important to note that three out of seven studies were conducted in China (Bai 2004; Fan 2006; Yongqin 2009) and this may be considered as a limitation to the generalisability of the results because of the differing cultural context of health and well-being. These three studies had no participants lost to follow-up.

The longest period of follow-up after completion of the intervention was six months (Duffy 2006). The remaining studies (Allison 2012; Bai 2004; Fan 2006; Humphris 2012; Katz 2004; Yongqin 2009) varied between three and four months. Therefore, the conclusions drawn from this review are only applicable for short and medium-term follow-up, with no evidence available for long-term follow-up.

\section{Quality of the evidence}

We rated only one study included in this review as being at an entirely low risk of bias (Allison 2012). The lack of important details on a number of the criteria in the other six studies (Bai 2004; Duffy 2006; Fan 2006; Humphris 2012; Katz 2004; Yongqin 2009) precluded an adequate assessment of their quality. Therefore, to employ a more robust judgement on the quality of the evidence, it is important for authors to provide clear, specific and relevant detail, especially on their method of participant selection. Figure 2 demonstrates that the overall risk of bias for four out of the seven studies is over $50 \%$. The blinding of participants to group allocation in such studies is especially challenging and in many instances may not be possible. Nonetheless, studies should introduce blinding of outcome assessment as a prerequisite. Blinding of outcome 
assessors was reported by Allison 2012, Fan 2006, Humphris 2012 and Katz 2004.

Other indicators of quality include power calculations to guide sample size. Only two studies (Allison 2012; Humphris 2012) report clearly that a power calculation was conducted, however the sample size required was not achieved in either study. Furthermore, many of the other included studies could be considered as underpowered to detect statistical differences between the experimental and control group.

A CONSORT flow chart was only present in two of the included studies (Allison 2012; Humphris 2012). Important information can be gleaned easily from these flowcharts, including poor accrual to such trials in this patient population. Allison 2012 highlights that $32 \%$ of participants approached to participate in the study refused to do so, leading to a concern regarding recruitment bias. The challenge of accrual of head and neck cancer patients to trials is a well-known phenomenon. A similar trend was reported by Duffy 2006, in that of the 439 eligible patients, 255 were not randomised due to refusal $(\mathrm{n}=179)$ or inability to contact $(\mathrm{n}=$ 76). Therefore, $42 \%$ of those eligible were recruited to this trial. With the exception of the three Chinese studies and the small pilot study by Katz 2004, attrition rates from the other trials were relatively high, varying between 14\% and 30\% (Allison $2012=$ $30 \%$, Duffy $2006=17 \%$, Humphris $2012=14 \%$ ).

The three studies conducted in China (Bai 2004; Fan 2006; Yongqin 2009) had their findings reported in Chinese and required translation. Data extraction forms were completed by one translator who was an author from the Cochrane ENT Disorder Group. Therefore, quality checks on data extraction from these three translated studies were not performed independently by a member of this review group.

\section{Potential biases in the review process}

This review took an inclusive approach to the term psychosocial intervention. Such a broad approach may have introduced heterogeneity, but it also led to a large number of references $(n=1035)$ being retrieved from the search, presenting the challenge of assessing and assimilating the evidence for relevant studies $(n=7)$. Due to the large number of references retrieved from the search for this patient population, we excluded grey literature such as dissertation theses and conference and poster abstracts. This again could introduce a degree of publication bias.

Some of the studies initially retrieved had mixed patient populations, including head and neck cancer survivors. Given that none of these studies had conducted subgroup analysis on patients with head and neck cancer, or following personal contact with the authors such data were not available, this review was limited to trials that solely recruited patients with head and neck cancer.

All the steps in the review process were conducted by two review authors (except for the data extraction forms for the three Chinese studies mentioned earlier). When necessary a third review author was consulted. Data input and analysis were carried out by the first author (CS) and checked by a second author (AN).

\section{Agreements and disagreements with other studies or reviews}

We identified one relevant systematic review (Luckett 2011). This systematic review included nine studies, of which two were RCTs, five were case controlled studies, one study was a pre-test post-test design and one study was post-test only with controls. No metaanalysis was conducted due to the heterogeneity in the type of interventions and outcome measures used. Based on a narrative synthesis, Luckett 2011 reported that there was some evidence for psychosocial interventions, with six out of the nine studies reporting at least one statistically significant benefit for the intervention versus control group. However, no studies found significant effects across all outcomes. In conclusion, Luckett 2011 provides a similar conclusion to this Cochrane review. In other words, the evidence for psychosocial intervention is limited due to the small number of studies, diversity of interventions evaluated and shortcomings in study designs and reporting.

Within the literature there is one further relevant descriptive review by Howren 2012. They appraised the evidence on several psychosocial and behavioural factors associated with head and neck cancer and included various psychosocial interventions. They concluded that several of the interventions appeared promising. However, they also noted that more rigorous and thorough testing is necessary given the lack of RCTs and the inherent methodological flaws in the small number of available studies. Furthermore, they highlighted the low accrual rates even for the briefest, least burdensome interventions.

\section{A U THORS' CONCLUSIONS}

\section{Implications for practice}

This review suggests that currently there is insufficient evidence to refute or support the effectiveness of psychosocial interventions for patients with head and neck cancer. This review was also unable to provide any evidence to support any specific type of psychosocial intervention for patients treated with head and neck cancer, in relation to theoretical perspective, duration, setting, mode of delivery or intensity.

Patients should be screened to target those at greatest risk of psychosocial difficulties, as currently there is no evidence to suggest a universal application of psychosocial intervention for all patients. The use of a tiered intervention approach in response to risk screening at key junctures in the cancer journey is recommended by the UK National Institute for Clinical Excellence (NICE) guidelines 
(NICE 2004) and the international guidelines for psycho-oncology (Coleman 2011).

\section{Implications for research}

Due to the small number of studies, which are generally not of high quality, the main conclusion of this review relates to how future research in this area should be conducted. There is a clear need for future intervention trials, but the ongoing challenge with conducting such studies is the relatively small number of patients available for accrual at any one cancer centre. To overcome this, a collaborative, multi-centred approach will be necessary. Furthermore, there is a need for a consensus on what type of intervention and delivery approach is most effective. Appraisal of the broader cancer literature to include systematic reviews on general or other site-specific cancer populations could help inform intervention design. It is important that interventions used in other cancer populations are modified for head and neck cancer patients, given the unique needs of this patient group, followed by further feasibility testing, prior to conducting larger randomised controlled trials (RCTs). Planning of such RCTs should include a power calculation to ensure adequate sample sizes, which will facilitate planning for recruitment.

There is a need for uniformity in outcome measures used to evaluate psychosocial interventions in head and neck cancer, thus enabling future meta-analyses to be conducted. Preference should be given to the European Organisation for Research and Treatment of Cancer Quality of Life Questionnaire (EORTC QLQ-C30) as a measure of quality of life. Modification of such validated measures must be discouraged as this again prevents data entry into meta-analyses.

There is currently much debate over which screening measure is most appropriate for anxiety and depression. Coyne and van Sonderena have heavily criticised the Hospital Anxiety and Depression Scale (HADS) for its psychometric qualities and suggest using other measures with cancer patients (Coyne 2012). This editorial was in response to the critical review of the factorial structure that has recently appeared in the literature (Cosco 2012). Subsequently, a comment by Doyle 2012 has pointed to a need for caution in the abandonment of the HADS, as it can still be useful to assess general distress. This is supported by Norton 2012. In addition, Norton and colleagues have conducted a strenuous meta-analysis of the HADS and found that although the factorial structure is not as clear as the originators of the scale suggested (two factors of depression and anxiety) the scale does behave quite consistently when a general distress factor is included - hence a three-factor model (Norton 2013).

Other outcome measures worth noting are the Patient Health Questionnaire (PHQ-9) (Kroenke 2001) and the Generalised
Anxiety Disorder seven-item scale (GAD-7) (Spitzer 2006) as measures for assessing general distress symptomology. The PHQ-9 is a short, easy to use depression module, which scores each of the nine Diagnostic and Statistical Manual of Mental Disorders (DSM-IV) criteria. Validity has been assessed against an independent, structured mental health professional interview. A PHQ-9 score $\geq 10$ had a sensitivity of $88 \%$ and a specificity of $88 \%$ for major depression (Kroenke 2001) and is worth considering as an outcome measure for depression in future studies. Whichever scale is deployed in research studies, there is an ongoing search for understanding of the construct validity and interpretation of the scores across age and demographic groups (Cameron 2012).

Consideration should also be given to the screening of individuals prior to recruitment. For example, if the premise of the intervention is to reduce fear of recurrence, participants should be screened using a suitably validated tool for this construct as part of the eligibility process, prior to accrual. This approach to eligibility according to an identified need is advocated and would avoid 'floor effects' of those with no expressed need, thus diluting the observed effects of psychosocial interventions. Mitchell 2012 also states that intervention programmes will have limited effect unless identified cases receive treatment which alters outcomes.

Another major consideration for future researchers within this field is the reporting of the findings. In order for RCTs to be appraised they should include detailed information about the intervention and its underlying theoretical premise. It is also necessary to describe the intervention setting, who delivered the intervention, its frequency and its intensity. In this field of psychosocial research it is particularly important that researchers are reminded that when publishing they provide sufficient information on method of randomisation, blinding etc., therefore allowing readers to make a judgement on the quality of the evidence. Also, due to the current financial constraints faced by many healthcare systems, there is an overwhelming need to involve health economists, to assess whether such interventions have cost benefits for providers of care to head and neck cancer survivors. In conclusion, there is a need for RCTs that are powered correctly, theoretically derived and have good endpoints and validated outcome.

\section{ACKNOW LEDGEMENTS}

Gemma Sandberg, Information Specialist/Trials Search Co-ordinator of the Cochrane ENT Disorders Group for her expert advice when developing the search strategy.

Jenny Bellorini, Managing Editor of the Cochrane ENT Disorder Group for her support and advice throughout this review. 


\section{R E F E R E N C E S}

\section{References to studies included in this review}

Allison 2012 \{unpublished data only\}

Allison PJ, Muller K, Edgar L, Nicolas JS, Feine JS, Franco EL. Randomized controlled trial of the effectiveness of a coping strategies intervention for people with head and neck cancer. Unpublished report for the Canadian Institutes of Health Research Award MCT-75475 2012:1-18.

Bai 2004 \{published data only\}

Bai S, Ma C, Liu Y, Xue W, Luo M, Ou Z. Effects of cognitive behavior intervention and cinesiateics on the quality of life of patients with nasopharyngeal carcinoma after radiotherapy. Chinese Journal of Clinical Rehabilitation 2004;8(29):6312-3.

Duffy 2006 \{published data only\} Duffy SA, Ronis DL, Valenstein M, Lambert MT, Fowler $\mathrm{KE}$, Gregory L, et al.Tailored smoking, alcohol and depression intervention for head and neck cancer patients. Cancer Epidemiology Biomarkers and Prevention 2006;15 (11):2203-8.

Fan 2006 \{published data only\}

Fan J, He X, Li J. Effects of combined interventional treatment on quality of life in patients with nasopharyngeal carcinoma. Chinese Journal of Clinical Rehabilitation 2006; 10(20):16-8.

Humphris 2012 \{unpublished data only\}

Humphris GM, Rogers SN. AFTER and beyond: cancer recurrence fears and a test of an intervention in oropharyngeal patients. Accepted for publication 2012.

Katz 2004 \{published data only\} Katz MR, Irish JC, Devins G. Development and pilot testing of a psychoeducational intervention for oral cancer patients. Psycho-Oncology 2004;13:642-53.

Yongqin 2009 \{published data only\}

Yongqin L, Xiupu Z. Influence of psychological and behavioural interventions on quality of life of postoperative patients after accepting total laryngectomy. Chinese Nursing Research 2009;23(9A):2287-90.

\section{References to studies excluded from this review}

de Maddalena \{published data only\}

de Maddalena H, Pfrang H. Improvement of communication behavior of laryngectomized and voicerehabilitated patients by a psychological training program. Head and Neck Oncology 1993;41(6):289-95.

Fiegenbaum 1981 \{published data only\}

Fiegenbaum W. A social training program for clients with facial disfigurations: a contribution to the rehabilitation of cancer patients. International Journal of Rehabilitation Research 1981;4(4):501-9.

Hammerlid 1999 \{published data only\} Hammerlid E, Persson L, Sulllivan M, Westin T. Qualityof-life effects of psychosocial intervention in patients with head and neck cancer. Otolaryngology - Head and Neck

Surgery 1999;120:507-16.

Head 2011 \{published data only\}

Head B, Keeney C, Studts JL, Khayat M, Bumpous J, Pfeifer $M$. Feasibility and acceptance of a telehealth intervention to promote symptom management during treatment for head and neck cancer. Journal of Supportive Oncology 2011;9(1): e1-e11.

McLachlan 2001 \{published data only\}

McLachlan S, Allenby A, Matthews J, Wirth A, Kissane

D, Bishop $M$, et al.Randomized trial of coordinated psychosocial interventions based on patient self-assessments versus standard care to improve the psychosocial functioning of patients with cancer. Journal of Clinical Oncology 2001; 19:4117-25.

Petruson 2003 \{published data only\} Petruson KM, Silander EM, Hammerlid EB. Effects of psychosocial intervention on quality of life in patients with head and neck cancer. Head and Neck 2003;25:576-84.

Semple 2009 \{published data only\}

Semple CJ, Dunwoody L, Kernohan WG, McCaughan

E. Development and evaluation of a problem-focused psychosocial intervention for patients with head and neck cancer. Supportive Care in Cancer 2009;17:379-88.

Sharma 2008 \{published data only\}

Sharma D, Nagarkar A, Jindal P, Kaur R, Gupta A. Personality changes and the role of counselling in the rehabilitation of patients with laryngeal cancer. Ear, Nose and Throat Journal 2008;87(8):E5.

Vakharia 2007 \{published data only\} Vakharia KT, Ali MJ, Wang ST. Quality of life impact of participation in a head and neck cancer support group. Otolaryngology - Head and Neck Surgery 2007;136:405-10.

van den Brink 2007 \{published data only\} van den Brink JL, Moorman PW, de Boer MF, Hop WC, Pruyn JF, Verwoerd CD, et al.Impact on quality of life of a telemedicine system supporting head and neck cancer patients: a controlled trial during the postoperative period at home. Journal of American Medical Informatics Association 2007;14(2):198-205.

Vilela 2006 \{published data only\} Vilela LA, Nicolau B, Mahmud S, Edgar L, Hier M, Black $\mathrm{M}$, et al.Comparison of psychosocial outcomes in head and neck cancer patients receiving a coping strategies intervention and control subjects receiving no intervention. Journal of Otolaryngology 2006;35(2):88-96.

\section{References to ongoing studies}

\section{de Leeuw \{published data only\}}

The effect of comprehensive counselling by a nurse specialist on depressive symptoms and quality of life: a prospective randomised study in patients with head and neck cancer. Ongoing study Dec 2003. 


\section{Kangas \{published data only\}}

Treatment of anxiety and depression in head and neck cancer patients. Ongoing study April 2007.

Verdonck-de Leeuw \{published and unpublished data\} Cost-effectiveness of a stepped care strategy to improve symptoms of depression or anxiety in patients treated for head and neck cancer or lung cancer. Ongoing study June 2009.

\section{Additional references}

\section{Aaronson 1993}

Aaronson NK, Ahmedzai S, Bergman B, Bullinger M, Cull A, Duez NJ, et al.The European Organisation for Research and Treatment of Cancer QLQ-C30: a quality-oflife instrument in international clinical trials in oncology. Journal of the National Cancer institute 1993;85(5):365-76.

Bessell 2009

Bessell A. Facing Up To Visible Difference: the Design and Evaluation of a New Computer-Based Psychosocial Intervention [Thesis]. Bristol, UK: University of the West of England, 2009.

\section{Cameron 2012}

Cameron IM, Crawford JR, Lawton K, Reid IC. Differential item functioning of the HADS and PHQ-9: an investigation of age, gender and educational background in a clinical UK primary care sample. Journal of Affective Disorders 2012 Dec 3 [Epub ahead of print].

\section{Coleman 2011}

Coleman N, Hession N, Connolly A. Psycho-oncology best practice guidelines and a service perspective: conceptualising the fit and towards bridging the gap. Irish Journal of Psychology 2011;32(1-2):72-89.

\section{Cosco 2012}

Cosco TD, Doyle F, Ward M, McGee H. Latent structure of the Hospital Anxiety And Depression Scale: a 10-year systematic review. Journal of Psychosomatic Research 2012; 72:180-4.

Coyne 2012

Coyne JC, van Sonderena E. No further research needed: abandoning the Hospital and Anxiety Depression Scale (HADS). Journal of Psychosomatic Research 2012;72:173-4.

\section{CRUK 2011}

Cancer Research UK. UK Oral cancer incidence statistics. http://info.cancerresearchuk.org/cancerstats/types/oral/ incidence/ (accessed 24 June 2011).

\section{Doyle 2012}

Doyle F, Cosco T, Conroy, R. Why the HADS is still important: reply to Coyne \& van Sonderen. Journal of Psychosomatic Research 2012;73:author reply 77-8.

Duffy 2007

Duffy SA, Ronis DL, Valenstein D, Fowler KE, Lambert MT, Bishop C, et al.Depressive symptoms, smoking, drinking, and quality of life among head and neck cancer patients. Psychosomatics 2007;48:142-8.

\section{Easterling 1989}

Easterling DV, Leventhal H. Contribution of concrete cognition to emotion: neutral symptoms as elicitors of worry about cancer. Journal of Applied Psychology 1989;74: 787-96.

Fawzy 1995

Fawzy FI, Fawzy NW, Arndt LA, Pasnau RO. Critical review of psychosocial interventions in cancer care. Archives of General Psychiatry 1995;52(2):100-13.

\section{Folkman 1980}

Folkman S, Lazarus RS. An analysis of coping in a middleaged community sample. Journal of Health and Social Behavior 1980;21(3):219-39.

Frampton 2001

Frampton M. Psychological distress in patients with head and neck cancer: review. British Journal of Oral and Maxillofacial Surgery 2001;39(1):67-70.

\section{Haman 2008}

Haman KL. Psychologic distress and head and neck cancer: part 1 - review of the literature. Journal of Supportive Oncology 2008;6(4):155-63.

\section{Handbook 2011}

Higgins JPT, Green S (editors). Cochrane Handbook for Systematic Reviews of Interventions Version 5.1.0 [updated March 2011]. The Cochrane Collaboration, 2011. Available from www.cochrane-handbook.org.

\section{Harrison 2009}

Harrison LB, Sessions RB, Hong WK. Head and Neck Cancer: a Multidisciplinary Approach. 3rd Edition. Philadelphia: Lippincott, Williams \& Wilkins, 2009.

\section{Holland 1982}

Holland JC. Psychological aspects of cancer. In: Holland JF, Frei E editor(s). Cancer Medicine. New York: Oxford University Press, 1982:1175-203.

\section{Holland 2005}

Holland JC, Reznik I. Pathways for psychosocial care of cancer survivors. Cancer 2005;104(Suppl 11):2624-37.

\section{Howren 2012}

Howren MB, Christensen AJ, Karnell LH, Funk GF. Psychological factors associated with head and neck cancer treatment and survivorship: evidence and opportunities for behavioral medicine. Journal of Consulting and Clinical Psychology 2012 Sep 10 [Epub ahead of print].

Humphris 2008

Humphris G, Ozakinci G. The AFTER intervention: a structured psychological approach to reduce fears of recurrence in patients with head and neck cancer. British Journal of Health Psychology 2008;13 (Part 2):223-30.

\section{Kroenke 2001}

Kroenke K, Spitzer RL, Williams JB. The PHQ-9: validity of a brief depression severity measure. Journal of General Internal Medicine 2001;16(9):606-13.

\section{Luckett 2011}

Luckett T, Britton B, Clover K, Rankin NM. Evidence for interventions to improve psychological outcomes in people 
with head and neck cancer: a systematic review of the literature. Supportive Care in Cancer 2011;19:871-81.

\section{Mitchell 2012}

Mitchell AJ, Meader N, Davies E, Clover K, Carter GL, Loscalzo MJ, et al.Meta-analysis of screening and case finding tools for depression in cancer: evidence based recommendations for clinical practice on behalf of the Depression in Cancer Care consensus group. Journal of Affective Disorders 2012;140:149-60.

\section{NICE 2004}

Guidance on Cancer Services: Improving Supportive and Palliative Care for Adults with Cancer. London: National Institute for Health and Care Excellence 2004.

\section{Norton 2012}

Norton S, Sacker A, Done J. Further research needed: a comment on Coyne and van Sonderen's call to abandon the Hospital Anxiety and Depression Scale. Journal of Psychosomatic Research 2012;73(1):75-6.

Norton 2013

Norton S, Cosco T, Doyle F, Done J, Sacker A. The Hospital Anxiety and Depression Scale: a meta confirmatory factor analysis. Journal of Psychosomatic Research 2012;74:74-81.

\section{Radloff 1977}

Radloff LS. The CES-D: a self-report depression scale for research in the general population. Applied Psychological Measurement 1977;1(3):385-401.

\section{RevMan 2012}

The Nordic Cochrane Centre, The Cochrane Collaboration. Review Manager (RevMan). 5.2. Copenhagen: The Nordic Cochrane Centre, The Cochrane Collaboration, 2012.

\section{Rosenberg 1965}

Rosenberg M. Society and the Adolescent Self-image. Princeton University Press, 1965.

\section{Semple 2004}

Semple CJ, Sullivan K, Dunwoody L, Kernohan WG. Psychosocial interventions for patients with head and neck cancer: past, present and future. Cancer Nursing 2004;27 (6):434-41

\section{Semple 2008}

Semple CJ, Dunwoody L, Kernohan WG, McCaughan E, Sullivan K. Changes and challenges to patients' lifestyles following treatment for head and neck cancer. Journal of Advanced Nursing 2008;63(1):85-93.

\section{Sheikh 1986}

Sheikh JI, Yesavage JA. Geriatric Depression Scale (GDS): recent evidence and development of a shorter version. Clinical Gerontology 1986;5(1/2):165-73.

\section{Spielberger 1980}

Spielberger CD, Gorsuch RL, Luchene RE. The State-Trait Anxiety Manual. California: Consulting Psychologists Press, 1980.

\section{Spitzer 2006}

Spitzer RL, Kroenke K, Williams JBW, Löwe B. A brief measure for assessing generalized anxiety disorder: the GAD-7. Archives of Internal Medicine 2006;166(10): 1092-7.

\section{Veer 2010}

Veer V, Kia S, Papesch M. Anxiety and depression in head and neck out-patients. Journal of Laryngology and Otology 2010;124(7):774-7.

\section{Watson 1989}

Watson M, Greer S, Bliss JM. Mental Adjustment to Cancer Scale User's Manual. Sutton, Surrey: Cancer Research Campaign Medical Research Group, Royal Marsden Hospital, 1989.

\section{Zigmond 1983}

Zigmond AS, Snaith RP. The Hospital Anxiety and Depression Scale. Acta Psychiatrica Scandinavica 1983;67: 361-70.

* Indicates the major publication for the study 


\section{CHARACTERISTICS OF STUDIES}

\section{Characteristics of included studies [ordered by study ID]}

\section{Allison 2012}

\begin{tabular}{l|l} 
Methods & $\begin{array}{l}\text { Multi-centred RCT } \\
\text { Conducted in Canada }\end{array}$ \\
\hline Participants & 61 participants \\
Age: mean 57.2 \\
Gender: male $61 \%(\mathrm{n}=37)$, female $39 \%(\mathrm{n}=24)$ \\
- Diagnosed with primary cancer of the head and neck region \\
- Screened for psychological distress prior to accrual and defined by scoring $>7$ on \\
the anxiety and/or depression scale of the Hospital Anxiety and Depression Scale \\
(HADS) \\
$\begin{array}{l}\text { Excluded if undergoing palliative or terminal care, had previous history of malignancy } \\
\text { affecting other parts of the body, diagnosed with depression and currently undergoing } \\
\text { any antidepressive therapy or living beyond } 90 \text { minutes travelling time by car/taxi from } \\
\text { recruitment site or unwilling to travel to recruitment hospital for intervention }\end{array}$
\end{tabular}

Interventions

Intervention group:

Description: psychoeducational programme consisting of problem-solving, relaxation techniques, cognitive coping skills, goal setting, communication social support and lifestyle factors

Duration: 2 or 3, 1 to 2 -hour sessions over a 4-week period. Provided with manual, plus audio material containing verbal instructions and music for relaxation section in CD or cassette

Delivered by trained therapists

Control group:

Description: attention placebo allowing participant to discuss cancer-related issues of their choice and informed of support services available at recruitment site (hospital) or local community services

Duration: 1 to 3, 1 to 2-hour sessions over a 4-week period

Delivered by trained therapist (same therapist as intervention group)

Outcomes

Baseline and 4 months post-randomisation

- Depression - HADS

- Coping - Ways of Coping Checklist

- Personality (optimism/pessimism) - Life Orientation Test

- Self esteem - Rosenberg Self-Esteem scale

\section{Risk of bias}

\section{Bias}

Random sequence generation (selection Low risk bias)

Computer-generated randomisation list used 
Allison 2012 (Continued)

\begin{tabular}{|c|c|c|}
\hline Allocation concealment (selection bias) & Low risk & $\begin{array}{l}\text { Generation of allocation sequence was per- } \\
\text { formed at a distant site by an individ- } \\
\text { ual who was otherwise independent of the } \\
\text { project }\end{array}$ \\
\hline $\begin{array}{l}\text { Blinding of participants and personnel } \\
\text { (performance bias) } \\
\text { All outcomes }\end{array}$ & Low risk & $\begin{array}{l}\text { Quote: "The study subjects were kept } \\
\text { blinded to their receiving the test or placebo } \\
\text { control interventions" }\end{array}$ \\
\hline $\begin{array}{l}\text { Blinding of outcome assessment (detection } \\
\text { bias) } \\
\text { All outcomes }\end{array}$ & Low risk & $\begin{array}{l}\text { Quote: "Blinding was also used for the re- } \\
\text { search assistant who collected the data" }\end{array}$ \\
\hline $\begin{array}{l}\text { Incomplete outcome data (attrition bias) } \\
\text { All outcomes }\end{array}$ & Low risk & Missing data clearly accounted for \\
\hline Selective reporting (reporting bias) & Low risk & All outcome data reported \\
\hline Other bias & Low risk & \\
\hline
\end{tabular}

\section{Bai 2004}

\begin{tabular}{ll}
\hline Methods & RCT \\
& Conducted in China \\
\hline Participants & 45 participants \\
& Age: mean 49.75 \\
& Gender: male $58 \%(\mathrm{n}=26)$, female $42 \%(\mathrm{n}=19)$ \\
& - Diagnosed with nasopharyngeal carcinoma \\
& - Karnofsky Performance Status $(\mathrm{KPS}) \geq 60$ \\
& - Received radical course of radiotherapy \\
& - No evidence of distant metastasis \\
& - No other chronic disease
\end{tabular}

Interventions

Intervention group:

Description: cognitive behaviour therapy and exercise (kinesiatric) intervention. Psychosocial component provided information on treatment, prognosis, cure and rehabilitation to include clarification of mistaken cognitions. Participants were encouraged to develop coping methods to include new life values, reduce tension and depression, establish positive life attitude and set hope for the future. Relaxation training and exercise activities of low to moderate intensity were set out for each participant

Duration: unclear

Delivered by: unclear

Control group:

Usual care

Outcomes

Assessed at 3 months after radiotherapy

- Quality of life - EORTC QLQ C30:

Physical function, role function, social function, emotional function, cognitive function,

Psychosocial interventions for patients with head and neck cancer (Review) 
general quality of life and 3 symptom items (fatigue, pain, nausea and vomiting)

Notes

Paper in Chinese and completion of data extraction form was arranged by editorial staff at Cochrane ENT Disorders Group

\section{Risk of bias}

\begin{tabular}{|c|c|c|}
\hline Bias & Authors' judgement & Support for judgement \\
\hline $\begin{array}{l}\text { Random sequence generation (selection } \\
\text { bias) }\end{array}$ & Unclear risk & $\begin{array}{l}\text { Randomised quoted in text; no description given of se- } \\
\text { quence generation }\end{array}$ \\
\hline Allocation concealment (selection bias) & Unclear risk & Inadequate information \\
\hline $\begin{array}{l}\text { Blinding of participants and personnel } \\
\text { (performance bias) } \\
\text { All outcomes }\end{array}$ & Unclear risk & Not mentioned \\
\hline $\begin{array}{l}\text { Blinding of outcome assessment (detection } \\
\text { bias) } \\
\text { All outcomes }\end{array}$ & High risk & $\begin{array}{l}\text { Quote: "the first and second authors and rehabilitative } \\
\text { physicians worked together to accomplish outcome assess- } \\
\text { ment" }\end{array}$ \\
\hline $\begin{array}{l}\text { Incomplete outcome data (attrition bias) } \\
\text { All outcomes }\end{array}$ & Low risk & No participants lost to follow-up \\
\hline Selective reporting (reporting bias) & Low risk & All outcome data reported \\
\hline Other bias & Low risk & None identified \\
\hline
\end{tabular}

Duffy 2006

\begin{tabular}{l|l}
\hline Methods & $\begin{array}{l}\text { Multi-centred RCT } \\
\text { Conducted in United States }\end{array}$ \\
\hline Participants & $\begin{array}{l}184 \text { participants } \\
\text { Age: mean } 57 \\
\text { Gender: male } 84 \%(\mathrm{n}=155) \text {, female } 16 \%(\mathrm{n}=29) \\
- \text { Screened positive for one or more of the following: smoking, alcohol use and } \\
\text { depression } \\
\bullet \text { Not pregnant } \\
\text { Excluded if non-English speaking, distant metastatic disease and/or were terminal and } \\
\text { unstable psychiatric/mental conditions such as suicidal ideation, acute psychosis, severe } \\
\text { alcohol dependence or dementia }\end{array}$ \\
\hline Interventions & $\begin{array}{l}\text { Intervention group: } \\
\text { Description: CBT delivered by telephone and workbook with pharmacologic interven- } \\
\text { tion, if required. CBT approaches emphasised goal-setting, self monitoring, analysed } \\
\text { behavioural antecedents, coping skills and social skills training. CBT sessions, workbook }\end{array}$ \\
\hline
\end{tabular}




\begin{tabular}{l} 
and pharmacologic interventions were tailored depending on health problem (smoking, \\
alcohol use and depression). CBT workbook had 4 sections: core section, tobacco tactics, \\
drinking decisions and mood management \\
$\begin{array}{l}\text { Duration: 45-minute nursing assessment and brief counselling, followed by } 9 \text { to } 11 \\
\text { telephone sessions of CBT } \\
\text { Delivered by nurses trained in CBT and supporting patients with tobacco and alcohol } \\
\text { problems } \\
\text { Control group: }\end{array}$ \\
$\begin{array}{l}\text { Description: enhanced usual care. Participants were referred as needed for smoking } \\
\text { cessation and/or alcohol treatment and/or psychiatric evaluation. Participants received a } \\
\text { handout for local, state and national resources } \\
\text { Duration: } 45-\text {-minute nursing assessment and brief counselling } \\
\text { Delivered by nurse }\end{array}$ \\
\hline $\begin{array}{l}\text { Baseline and } 6 \text { months post-intervention } \\
\text { - Smoking status (no tool) } \\
\text { - Alcohol use: Alcohol Use Disorder Identification Test (AUDIT) } \\
\text { - Depression: Geriatric Depression Scale-Short Form }\end{array}$ \\
\hline $\begin{array}{l}\text { Funding from the Department of Veterans Affairs IIR98-500, GlaxoSmithKline through } \\
\text { the Managed Care Forum and the NIH through the University of Michigan's Head and } \\
\text { Neck Specialized Programs for Research Excellence grant 1P50 CA97248 }\end{array}$ \\
\hline Notes
\end{tabular}

\section{Risk of bias}

\begin{tabular}{l|l|l}
\hline Bias & Authors' judgement & Support for judgement \\
\hline $\begin{array}{l}\text { Random sequence generation (selection } \\
\text { bias) }\end{array}$ & Unclear risk & $\begin{array}{l}\text { Randomised quoted in text; no description } \\
\text { given of sequence generation }\end{array}$ \\
\hline $\begin{array}{l}\text { Allocation concealment (selection bias) } \\
\begin{array}{l}\text { Blinding of participants and personnel } \\
\text { (performance bias) } \\
\text { All outcomes }\end{array}\end{array}$ & Unclear risk & Not mentioned \\
\hline
\end{tabular}

Blinding of outcome assessment (detection Unclear risk

Not mentioned

bias)

All outcomes

\begin{tabular}{l|l|l}
$\begin{array}{l}\text { Incomplete outcome data (attrition bias) } \\
\text { All outcomes }\end{array}$ & Low risk & Missing data accounted for \\
\hline
\end{tabular}

All outcomes

\begin{tabular}{|c|c|c|}
\hline Selective reporting (reporting bias) & High risk & $\begin{array}{l}\text { Only dichotomous data reported for AU- } \\
\text { DIT and CES-D scales }\end{array}$ \\
\hline Other bias & High risk & $\begin{array}{l}\text { Funding from GlaxoSmithKline who pro- } \\
\text { duce both nicotine patch and bupropion } \\
\text { which are part of the pharmacologic man- }\end{array}$ \\
\hline
\end{tabular}


Duffy 2006 (Continued)

agement protocol for tailored intervention (Appendix A)

Fan 2006

\begin{tabular}{|c|c|}
\hline Methods & $\begin{array}{l}\text { RCT } \\
\text { Conducted in China }\end{array}$ \\
\hline Participants & $\begin{array}{l}72 \text { participants } \\
\text { Age: mean } 51.95 \\
\text { Gender: not recorded } \\
\text { - Diagnosed with nasopharyngeal carcinoma } \\
\text { Excluded if in terminal phase of illness, history of mental illness or could not understand } \\
\text { the content of the questionnaires }\end{array}$ \\
\hline Interventions & $\begin{array}{l}\text { Intervention group: } \\
\text { Description: systematic mass anticancer education over a } 2 \text {-year period focusing on body } \\
\text { function, mental well-being and social reintegration. The } 3 \text { components encompassed: } \\
\text { 1) physical function rehabilitation - lecture on the pathogenic mechanism, treatment, } \\
\text { follow-up and prognosis of nasopharyngeal carcinoma, and regular lectures on rehabili- } \\
\text { tation and exercise training; 2) mental well-being - lecture on mental health and group } \\
\text { activities on cognitive psychological techniques; } 3 \text { ) social reintegration - small group } \\
\text { activities exchanging roles, reassessing responsibilities of society and family, and class on } \\
\text { health education and rehabilitation } \\
\text { Duration: } 2 \text { years } \\
\text { Physicians, nurses and medical students were trained to deliver the intervention } \\
\text { Control group: }\end{array}$ \\
\hline Outcomes & $\begin{array}{l}\text { Baseline and following the intervention (duration of intervention: } 2 \text { years) } \\
\text { - Quality of life - the statistical survey table of vital quality of patients with } \\
\text { carcinoma of the healing stage, designed after the EORTC QLQ-C } 30 \text { with the } \\
\text { following subscales: } \\
\text { Physical function, mental health, social function, general quality of life, symptom and } \\
\text { side effects and special side effects }\end{array}$ \\
\hline Notes & $\begin{array}{l}\text { Supported by the Basic Research Program of Application in Sichuan Province, No. } \\
01 S Y 051-46 \\
\text { Paper in Chinese and data extraction was arranged by editorial staff at the Cochrane } \\
\text { ENT Disorders Group }\end{array}$ \\
\hline
\end{tabular}

\section{Risk of bias}

\section{Bias}

Random sequence generation (selection Unclear risk bias)

\section{Support for judgement}

Randomised quoted in text; no description given of sequence generation 
Fan 2006 (Continued)

\begin{tabular}{l|ll}
\hline Allocation concealment (selection bias) & Unclear risk & Inadequate information \\
\hline $\begin{array}{l}\text { Blinding of participants and personnel } \\
\text { (performance bias) } \\
\text { All outcomes }\end{array}$ & Unclear risk & Not mentioned \\
\hline $\begin{array}{l}\text { Blinding of outcome assessment (detection } \\
\text { bias) } \\
\text { All outcomes }\end{array}$ & Low risk & $\begin{array}{l}\text { Quote: "double-blind investigation was conducted on pa- } \\
\text { tients by investigators" }\end{array}$ \\
\hline $\begin{array}{l}\text { Incomplete outcome data (attrition bias) } \\
\text { All outcomes }\end{array}$ & Low risk & No participants lost to follow-up \\
\hline $\begin{array}{l}\text { Selective reporting (reporting bias) } \\
\text { Other bias }\end{array}$ & Low risk & All outcome data reported \\
\hline
\end{tabular}

Humphris 2012

\begin{tabular}{|c|c|}
\hline Methods & $\begin{array}{l}\text { RCT } \\
\text { Conducted in United Kingdom }\end{array}$ \\
\hline Participants & $\begin{array}{l}77 \text { participants ( } 87 \text { participants randomised, } 10 \text { lost to follow-up) } \\
\text { Age: mean } 59 \\
\text { Gender: male } 71 \% \text {, female } 29 \% \\
\text { - Diagnosed with primary orofacial cancer } \\
\text { - Age range of } 18 \text { to } 75 \text { years } \\
\text { Excluded if psychotic, threatened to commit suicide or severe intellectual deficit }\end{array}$ \\
\hline Interventions & $\begin{array}{l}\text { Intervention group: } \\
\text { Description - time-limited, theory driven, structured, individualised psychological in- } \\
\text { tervention based on a cognitive behavioural model of self regulation to address fears of } \\
\text { recurrence. Patient is assessed at first session and intervention tailored. Invited to explore } \\
\text { illness belief and behaviours. Relaxation practised in sessions. Caregivers can be incor- } \\
\text { porated in programme if desired by patient } \\
\text { Duration - } 6 \text { weekly sessions. Length of each intervention session = } 30 \text { to } 50 \text { minutes } \\
\text { Delivered by trained specialist nurse who followed a standardised manual (AFTER - } \\
\text { adjustment to fears, threats and expectation of recurrence) } \\
\text { Control group: }\end{array}$ \\
\hline
\end{tabular}

Outcomes

Data collected 3, 7, 11 and 15 months following treatment. Intervention was delivered during the interval between 7 and 11-month assessment. Baseline was considered as 7 months, end of intervention as 11 months and 14 months as 4 months post-intervention

- Depression - HADS

- Worry - Worry of Cancer Scale (WOC)

- Adjustment to cancer - Mental Adjustment to Cancer Scale

- Quality of life - EORTC QLQ-C30 v 2, which consists of 5 functional scales 
Humphris 2012 (Continued)

(physical, role, cognitive, emotional and social), a separate global quality of life scale and 3 symptom scales (fatigue, pain and nausea and vomiting)

- Disease-specific quality of life - EORTC QLQ H\&N35

Notes Supported by CR-UK CP1031/0102

\section{Risk of bias}

\begin{tabular}{|c|c|c|}
\hline Bias & Authors' judgement & Support for judgement \\
\hline $\begin{array}{l}\text { Random sequence generation (selection } \\
\text { bias) }\end{array}$ & Low risk & $\begin{array}{l}\text { Quote: "computer-generated random number } \\
\text { sequence" }\end{array}$ \\
\hline
\end{tabular}

Allocation concealment (selection bias) Low risk

Quote: "she subsequently made contact with the study administrator who picked the next numbered envelope from the computer-generated random number sequence (independently prepared by the faculty statistician)"

Blinding of participants and personnel Unclear risk Insufficient information (performance bias)

All outcomes

Blinding of outcome assessment (detection Low risk bias)

All outcomes

Quote: "assessor was blind to group assignment", "post-intervention data blind to therapist”

Incomplete outcome data (attrition bias) Low risk

All outcomes

10 patients lost to follow-up and rationale given in section "adherence to study design"

\begin{tabular}{l|l|l} 
Selective reporting (reporting bias) & Unclear risk & Narrative only on quality of life data
\end{tabular}

Other bias

Unclear risk

Following randomisation 5 participants in the intervention group declined intervention but outcome data included and remain as part of intervention arm in study

Katz 2004

\begin{tabular}{|c|c|}
\hline Methods & $\begin{array}{l}\text { RCT } \\
\text { Conducted in Canada }\end{array}$ \\
\hline Participants & $\begin{array}{l}19 \text { participants } \\
\text { Age: mean } 56.6 \\
\text { Gender: male } 63 \%(\mathrm{n}=14) \text {, female } 37 \%(\mathrm{n}=5) \\
\text { - Newly diagnosed, untreated oral cavity cancer } \\
\text { - Proposed curative surgical treatment } \\
\text { - Absence of significant cognitive impairment }\end{array}$ \\
\hline
\end{tabular}


- Minimum of Grade 8 education

Interventions
Intervention group:
Description: psychoeducational intervention delivered in verbal and written format.
The psychoeducational booklet $(95$ pages) was disseminated in 2 parts: a) preparing
for surgery and the postoperative course and b) preparing for your return home. This
booklet covered information about oral cancer and its treatment and effective coping
strategies. The content was presented by a research nurse and patients were encouraged
to ask questions and express concerns
Duration: 2 brief supportive contacts ( 60 to 90 minutes). First supportive contact was
pre-operatively, via telephone. Second supportive contact was postoperatively, via face-
to-face contact
Delivered by a research nurse
Control group:
Usual care

Outcomes

Baseline (pre-operative) and 3-months post-discharge

Knowledge questionnaire

Disfigurement: Observer-Rated Disfigurement Scale

Alcohol use: SMAST-13 Alcoholism

Social Support: MOS Social Support Survey (perceived social support)

Recent stressful life events (composite checklist)

Self efficacy: Stanford Inventory of Cancer Patients Adjustment (SICPA)

Anxiety: State-Trait Anxiety Inventory (STAI)

Depression: Center for Epidemiologic Studies-Depression scale (CES-D)

Well-being: Affect Balance Scale

Life satisfaction: Atkinson Life Happiness Rating Scale

Illness intrusiveness: Illness Intrusiveness Rating Scale

Quality of life: EORTC QLQ-C30

Body image (unpublished by authors)

Funded by National Cancer Institute of Cancer (grant \#007096)

Risk of bias

Bias

Authors' judgement

Support for judgement

Random sequence generation (selection Low risk

Quote: "Random numbers table"

bias)

\begin{tabular}{|c|c|c|}
\hline Allocation concealment (selection bias) & Unclear risk & No detail provided \\
\hline $\begin{array}{l}\text { Blinding of participants and personnel } \\
\text { (performance bias) } \\
\text { All outcomes }\end{array}$ & Unclear risk & $\begin{array}{l}\text { Blinding of participants or personnel delivering interven- } \\
\text { tion not mentioned. Only reference to the research nurse } \\
\text { delivering the intervention being blind to the results of } \\
\text { all assessments }\end{array}$ \\
\hline
\end{tabular}

Psychosocial interventions for patients with head and neck cancer (Review) 


\section{Katz 2004 (Continued)}

\begin{tabular}{l|l}
$\begin{array}{l}\text { Blinding of outcome assessment (detection } \\
\text { bias) }\end{array}$ & Low risk \\
All outcomes & $\begin{array}{l}\text { Quote: "The study assessment packages were adminis- } \\
\text { tered by the research coordinator who was not involved } \\
\text { in the delivery of the intervention" }\end{array}$
\end{tabular}

\begin{tabular}{lll}
\hline $\begin{array}{l}\text { Incomplete outcome data (attrition bias) } \\
\text { All outcomes }\end{array}$ & Low risk & No participants lost to follow-up \\
\hline Selective reporting (reporting bias) & Low risk & All outcome data reported \\
\hline Other bias & High risk & Multiple test with a very small sample size \\
\hline
\end{tabular}

\section{Yongqin 2009}

\begin{tabular}{l|l}
\hline Methods & $\begin{array}{l}\text { RCT } \\
\text { Conducted in China }\end{array}$ \\
\hline Participants & $\begin{array}{l}84 \text { participants } \\
\text { Age: mean } 43.2 \\
\text { Gender: male } 84.5 \%(\mathrm{n}=71) \text {, female } 15.5 \%(\mathrm{n}=13) \\
-\quad \text { Biopsy proven laryngeal or hypopharyngeal carcinoma } \\
\text { - Having undergone a laryngectomy }\end{array}$ \\
$\begin{array}{l}\text { Excluded if diagnosed with a second cancer following laryngectomy, had local or distant } \\
\text { metastasis } 6 \text { months after surgery, lack of follow-up data (more than } 50 \%) \text { or failed to } \\
\text { attend follow-up }\end{array}$ \\
\hline $\begin{array}{l}\text { Interventions } \\
\text { Intervention group: }\end{array}$ \\
$\begin{array}{l}\text { Description: CBT. Cognitive therapy included understanding the cancer, learning how } \\
\text { to cope and ensuring the patients had social support. Behavioural therapy included new } \\
\text { ways of communication, self management of stoma and patients were encouraged to } \\
\text { take part in group activity, recreation and sports but maintain balance between rest and } \\
\text { activities that would promote rehabilitation } \\
\text { Duration: } 2 \text { years } \\
\text { Delivered by: unclear } \\
\text { Control group: } \\
\text { Usual care }\end{array}$ \\
\hline
\end{tabular}

Outcomes

Baseline and following the intervention (duration of intervention 2 years)

Quality of life: the quality of life table for Washington medical college students - Chinese version ( $v 2$ ) with the following subscales: pain, appearance, instrumental activities of daily living, recreation, work, chew, swallow, speaking and communication and shoulder function

Notes

Paper in Chinese and data extraction was arranged by editorial staff at the Cochrane ENT Disorders Group

\section{Risk of bias}

\section{Support for judgement}




\section{Yongqin 2009 (Continued)}

\begin{tabular}{l|l|l}
$\begin{array}{l}\text { Random sequence generation (selection } \\
\text { bias) }\end{array}$ & Unclear risk & $\begin{array}{l}\text { Randomised quoted in text; no description given of se- } \\
\text { quence generation }\end{array}$ \\
\hline $\begin{array}{l}\text { Allocation concealment (selection bias) } \\
\begin{array}{l}\text { Blinding of participants and personnel } \\
\text { (performance bias) } \\
\text { All outcomes }\end{array}\end{array}$ & Unclear risk & Not mentioned \\
\hline $\begin{array}{l}\text { Blinding of outcome assessment (detection } \\
\text { bias) } \\
\text { All outcomes }\end{array}$ & Unclear risk & Not mentioned \\
\hline $\begin{array}{l}\text { Incomplete outcome data (attrition bias) } \\
\text { All outcomes }\end{array}$ & Low risk & No participants reported as lost to follow-up \\
\hline \begin{tabular}{l} 
Selective reporting (reporting bias) \\
\hline
\end{tabular} & Low risk & All outcome data reported \\
\hline
\end{tabular}

AUDIT: Alcohol Use Disorder Identification Test

CBT: cognitive behavioural therapy

CES-D: Centre for Epidemiologic Studies Depression Scale

EORTC: European Organisation for Research and Treatment of Cancer

ENT: ear, nose and throat

HADS: Hospital Anxiety and Depression Scale

NIH: National Institutes of Health

QLQ: Quality of Life Questionnaire

RCT: randomised controlled tria

\section{Characteristics of excluded studies [ordered by study ID]}

\begin{tabular}{|c|c|}
\hline Study & Reason for exclusion \\
\hline de Maddalena & $\begin{array}{l}\text { ALLOCATION } \\
\text { Randomised } \\
\text { PARTICIPANTS } \\
\text { Included patients with head and neck cancer } \\
\text { INTERVENTION } \\
\text { Patients received psychological communication training } \\
\text { OUTCOMES } \\
\text { Did not use a validated outcome measure }\end{array}$ \\
\hline Fiegenbaum 1981 & $\begin{array}{l}\text { ALLOCATION } \\
\text { Not randomised }\end{array}$ \\
\hline
\end{tabular}


(Continued)

\begin{tabular}{|c|c|}
\hline Hammerlid 1999 & $\begin{array}{l}\text { ALLOCATION } \\
\text { Not randomised }\end{array}$ \\
\hline Head 2011 & $\begin{array}{l}\text { ALLOCATION } \\
\text { Randomised } \\
\text { PARTICIPANTS } \\
\text { Included patients with head and neck cancer } \\
\text { INTERVENTION } \\
\text { No face-to-face contact. Focus was symptom control and not a psychosocial intervention } \\
\text { OUTCOMES } \\
\text { No control data published to date (data sought but not received) }\end{array}$ \\
\hline McLachlan 2001 & $\begin{array}{l}\text { ALLOCATION } \\
\text { Randomised } \\
\text { PARTICIPANTS } \\
\text { Included patients with head and neck cancer. No separate subgroup analysis for patients with head and neck } \\
\text { cancer }(\mathrm{n}=96) \text { (data sought but not received) }\end{array}$ \\
\hline Petruson 2003 & $\begin{array}{l}\text { ALLOCATION } \\
\text { Not randomised }\end{array}$ \\
\hline Semple 2009 & $\begin{array}{l}\text { ALLOCATION } \\
\text { Not randomised }\end{array}$ \\
\hline Sharma 2008 & $\begin{array}{l}\text { ALLOCATION } \\
\text { Randomised } \\
\text { PARTICIPANTS } \\
\text { Included patients with head and neck cancer } \\
\text { INTERVENTION } \\
\text { Patients received psychosocial intervention - individualised counselling } \\
\text { OUTCOMES } \\
\text { Personality Trait Inventory - not predefined primary or secondary outcome measure }\end{array}$ \\
\hline Vakharia 2007 & $\begin{array}{l}\text { ALLOCATION } \\
\text { Not randomised }\end{array}$ \\
\hline van den Brink 2007 & $\begin{array}{l}\text { ALLOCATION } \\
\text { Not randomised - quasi-experimental design (based on hospital location) } \\
\text { PARTICIPANTS } \\
\text { Included patients with head and neck cancer } \\
\text { INTERVENTION } \\
\text { Electronic health information - no face-to-face contact }\end{array}$ \\
\hline Vilela 2006 & $\begin{array}{l}\text { ALLOCATION } \\
\text { Not randomised }\end{array}$ \\
\hline
\end{tabular}

Psychosocial interventions for patients with head and neck cancer (Review) 
Characteristics of ongoing studies [ordered by study ID]

\section{de Leeuw}

Trial name or title The effect of comprehensive counselling by a nurse specialist on depressive symptoms and quality of life: a prospective randomised study in patients with head and neck cancer

\begin{tabular}{ll}
\hline Methods & RCT, parallel group \\
\hline Participants & 154 (target number) \\
\hline Interventions & $\begin{array}{l}\text { Comprehensive counselling by a nurse specialist to help the patient deal with physical symptoms and impair- } \\
\text { ments, to reduce emotional distress and improve morale, coping and sense of control } \\
6 \text { sessions over a 1-year period }\end{array}$ \\
\hline Outcomes & CES-D, EORTC QLQ-C30, EORTC QLQ-HN35, Worry of Cancer Scale, Uncertainty in Illness Scale \\
\hline Starting date & Dec 2003 \\
\hline Contact information & Dr JRL de Leeuw \\
\hline Notes & Recruitment is possibly completed. Additional information sought but not obtained on trial status \\
\hline
\end{tabular}

\section{Kangas}

Trial name or title Treatment of anxiety and depression in head and neck cancer patients

\begin{tabular}{ll}
\hline Methods & RCT, parallel group \\
\hline Participants & 160 (target number) \\
\hline Interventions & $\begin{array}{l}\text { CBT comprising relaxation, stress management and coping skills training } \\
6 \text { weekly sessions, face-to-face during radiotherapy and a booster session of CBT 1 month following comple- } \\
\text { tion of radiotherapy }\end{array}$ \\
\hline Outcomes & $\begin{array}{l}\text { Clinician Administered PTSD Scale, Beck Depression Inventory, STAI, FACT, Brief COPE, treatment cred- } \\
\text { ibility scale to assess individuals' beliefs about treatment efficacy }\end{array}$ \\
\hline Starting date & April 2007 \\
\hline Contact information & Dr Maria Kangas \\
\hline Notes & $\begin{array}{l}\text { Recruitment is possibly completed. Additional information sought from primary author but not obtained on } \\
\text { trial status }\end{array}$ \\
\hline
\end{tabular}


Verdonck-de Leeuw

Trial name or title Cost-effectiveness of a stepped care strategy to improve symptoms of depression or anxiety in patients treated for head and neck cancer or lung cancer

\begin{tabular}{ll}
\hline Methods & RCT, parallel group \\
\hline Participants & 176 (target number) \\
\hline Interventions & $\begin{array}{l}\text { Stepped care model with 4 evidence-based steps: 1) watchful waiting, 2) internet-based self help, 3) problem- } \\
\text { solving therapy applied by a nurse, 4) specialised psychological intervention and/or antidepressant medication } \\
\text { Control group: usual care }\end{array}$ \\
\hline Outcomes & $\begin{array}{l}\text { HADS, EORTC QLQ-C30, EORTC QLQ-HN35, EORTC QLQ-LC13, EORTC QLQ-PATSAT, health } \\
\text { care utilisation and work loss (TIC-P and PRODISQ modules) }\end{array}$ \\
\hline Starting date & June 2009 \\
\hline Contact information & Dr IM Verdonck-de Leeuw \\
\hline Notes & \\
\hline
\end{tabular}

CBT: cognitive behavioural therapy

CES-D: Centre for Epidemiologic Studies Depression Scale

EORTC: European Organisation for Research and Treatment of Cancer

FACT: Functional Assessment of Cancer Therapy

HADS: Hospital Anxiety and Depression Scale

PATSAT: patient satisfaction with care

PTSD: Post-Traumatic Stress Disorder

QLQ: Quality of Life Questionnaire

RCT: randomised controlled trial

STAI: State-Trait Anxiety Inventory 
DATA AND ANALYSES

\section{Comparison 1. Intervention versus control}

\begin{tabular}{|c|c|c|c|c|}
\hline Outcome or subgroup title & $\begin{array}{l}\text { No. of } \\
\text { studies }\end{array}$ & $\begin{array}{c}\text { No. of } \\
\text { participants }\end{array}$ & Statistical method & Effect size \\
\hline $\begin{array}{l}1 \text { EORTC QLQ C30 - Physical } \\
\text { function (medium-term } \\
\text { follow-up) }\end{array}$ & 3 & 138 & Mean Difference (IV, Random, 95\% CI) & $6.28[-10.05,22.60]$ \\
\hline $\begin{array}{l}2 \text { EORTC QLQ C30 - Emotional } \\
\text { function (medium-term } \\
\text { follow-up) }\end{array}$ & 3 & 138 & Mean Difference (IV, Random, 95\% CI) & $2.54[-4.81,9.89]$ \\
\hline $\begin{array}{l}3 \text { EORTC QLQ C30 - Social } \\
\text { function (medium-term } \\
\text { follow-up) }\end{array}$ & 3 & 138 & Mean Difference (IV, Random, 95\% CI) & $-2.36[-11.36,6.65]$ \\
\hline $\begin{array}{l}4 \text { EORTC QLQ C30 - Role } \\
\text { function (medium-term } \\
\text { follow-up) }\end{array}$ & 3 & 138 & Mean Difference (IV, Random, 95\% CI) & $-1.43[-7.31,4.46]$ \\
\hline $\begin{array}{l}5 \text { EORTC QLQ C30 - Global } \\
\text { quality of life (medium-term } \\
\text { follow-up) }\end{array}$ & 3 & 138 & Mean Difference (IV, Random, 95\% CI) & $-2.57[-13.54,8.40]$ \\
\hline $\begin{array}{l}6 \text { EORTC QLQ C30 - Cognitive } \\
\text { function (medium-term } \\
\text { follow-up) }\end{array}$ & 3 & 138 & Mean Difference (IV, Random, 95\% CI) & $-4.23[-12.48,4.03]$ \\
\hline $\begin{array}{l}7 \text { EORTC QLQ C30 - Cognitive } \\
\text { function (end of intervention) }\end{array}$ & 3 & 141 & Mean Difference (IV, Random, 95\% CI) & $2.78[-4.82,10.37]$ \\
\hline $\begin{array}{l}8 \text { EORTC QLQ C30 - Physical } \\
\text { function (end of intervention) }\end{array}$ & 3 & 141 & Mean Difference (IV, Random, 95\% CI) & $6.74[-2.24,15.71]$ \\
\hline $\begin{array}{l}9 \text { EORTC QLQ C30 - Emotional } \\
\text { function (end of intervention) }\end{array}$ & 3 & 141 & Mean Difference (IV, Random, 95\% CI) & $4.15[-3.60,11.89]$ \\
\hline $\begin{array}{l}10 \text { EORTC QLQ C30 - Social } \\
\text { function (end of intervention) }\end{array}$ & 3 & 141 & Mean Difference (IV, Random, 95\% CI) & $-3.03[-12.04,5.98]$ \\
\hline $\begin{array}{l}11 \text { EORTC QLQ C30 - Role } \\
\text { function (end of intervention) }\end{array}$ & 3 & 141 & Mean Difference (IV, Random, 95\% CI) & $1.31[-4.85,7.47]$ \\
\hline $\begin{array}{l}12 \text { EORTC QLQ C30 - } \\
\text { Global quality of life (end of } \\
\text { intervention) }\end{array}$ & 3 & 141 & Mean Difference (IV, Random, 95\% CI) & $1.23[-5.82,8.27]$ \\
\hline $\begin{array}{l}13 \text { Anxiety (medium-term } \\
\text { follow-up } 3 \text { to } 4 \text { months) }\end{array}$ & 3 & 157 & Std. Mean Difference (IV, Random, 95\% CI) & $-0.09[-0.40,0.23]$ \\
\hline 14 Anxiety (end of intervention) & 3 & 157 & Std. Mean Difference (IV, Random, 95\% CI) & $-0.09[-0.41,0.23]$ \\
\hline $\begin{array}{l}15 \text { Depression (medium term } \\
\text { follow-up } 3 \text { to } 6 \text { months) }\end{array}$ & 4 & 335 & Std. Mean Difference (IV, Random, 95\% CI) & $-0.03[-0.24,0.19]$ \\
\hline $\begin{array}{l}16 \text { Depression (end of } \\
\text { intervention) }\end{array}$ & 4 & 335 & Std. Mean Difference (IV, Random, 95\% CI) & $-0.00[-0.22,0.21]$ \\
\hline
\end{tabular}

Psychosocial interventions for patients with head and neck cancer (Review) 
Analysis I.I. Comparison I Intervention versus control, Outcome I EORTC QLQ C30 - Physical function (medium-term follow-up).

Review: Psychosocial interventions for patients with head and neck cancer

Comparison: I Intervention versus control

Outcome: I EORTC QLQ C30 - Physical function (medium-term follow-up)

\begin{tabular}{|c|c|c|c|c|c|c|c|}
\hline \multirow[t]{2}{*}{ Study or subgroup } & \multirow{2}{*}{$\begin{array}{r}\text { Intervention } \\
\mathrm{N}\end{array}$} & \multicolumn{3}{|c|}{ Control } & \multirow{2}{*}{$\begin{array}{c}\text { Mean } \\
\text { Difference } \\
\text { IV,Random,95\% Cl }\end{array}$} & \multirow[t]{2}{*}{ Weight } & \multirow{2}{*}{$\begin{array}{r}\text { Mean } \\
\text { Difference } \\
\text { IV,Random,95\% Cl }\end{array}$} \\
\hline & & Mean(SD) & $N$ & Mean(SD) & & & \\
\hline Bai 2004 & 24 & $64.21(26.14)$ & 21 & $53.25(18.64)$ & 7 & $39.4 \%$ & $10.96[-2.19,24.11]$ \\
\hline Humphris 2012 & 44 & $64(30.3)$ & 30 & $72(30.4)$ & $\Rightarrow$ & $37.9 \%$ & $-8.00[-22.09,6.09]$ \\
\hline Katz 2004 & 10 & $87(22.4)$ & 9 & $65(33.4)$ & $\square$ & $22.7 \%$ & $22.00[-3.86,47.86]$ \\
\hline
\end{tabular}

Total (95\% CI)

$78 \quad 60$

Heterogeneity: $\mathrm{Tau}^{2}=131.24 ; \mathrm{Chi}^{2}=5.71, \mathrm{df}=2(\mathrm{P}=0.06) ; \mathrm{I}^{2}=65 \%$

Test for overall effect: $Z=0.75(P=0.45)$

Test for subgroup differences: Not applicable

\begin{tabular}{|c|c|c|c|c|}
\hline-100 & -50 & 0 & 50 & 100 \\
\hline
\end{tabular}


Analysis I.2. Comparison I Intervention versus control, Outcome 2 EORTC QLQ C30 - Emotional function (medium-term follow-up).

Review: Psychosocial interventions for patients with head and neck cancer

Comparison: I Intervention versus control

Outcome: 2 EORTC QLQ C30 - Emotional function (medium-term follow-up)

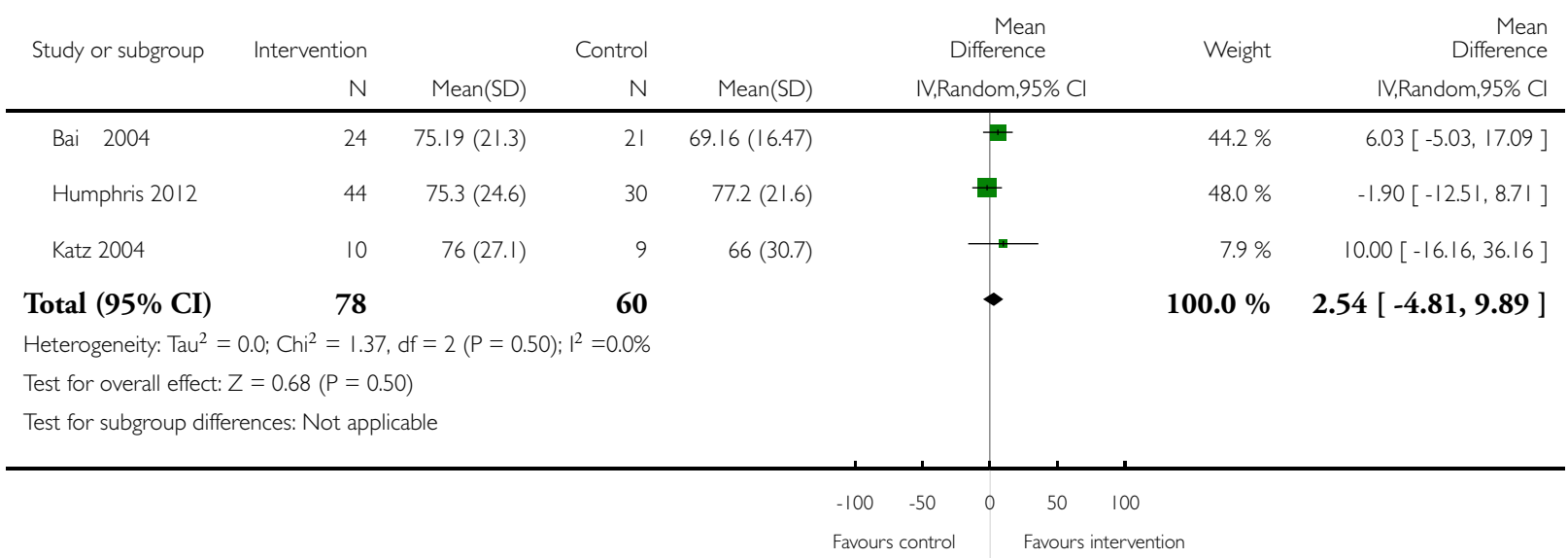




\section{Analysis I.3. Comparison I Intervention versus control, Outcome 3 EORTC QLQ C30 - Social function}

(medium-term follow-up).

Review: Psychosocial interventions for patients with head and neck cancer
Comparison: I Intervention versus control
Outcome: 3 EORTC QLQ C30 - Social function (medium-term follow-up)

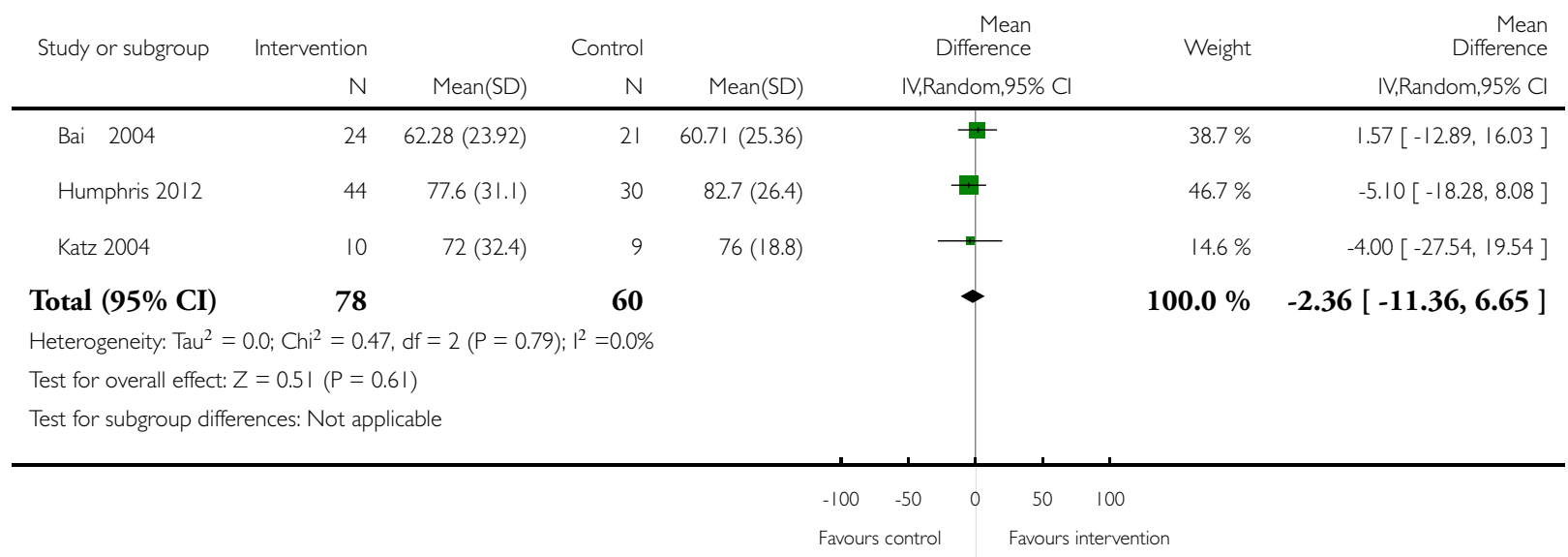


Analysis I.4. Comparison I Intervention versus control, Outcome 4 EORTC QLQ C30 - Role function (medium-term follow-up).

Review: Psychosocial interventions for patients with head and neck cancer
Comparison: I Intervention versus control
Outcome: 4 EORTC QLQ C30 - Role function (medium-term follow-up)

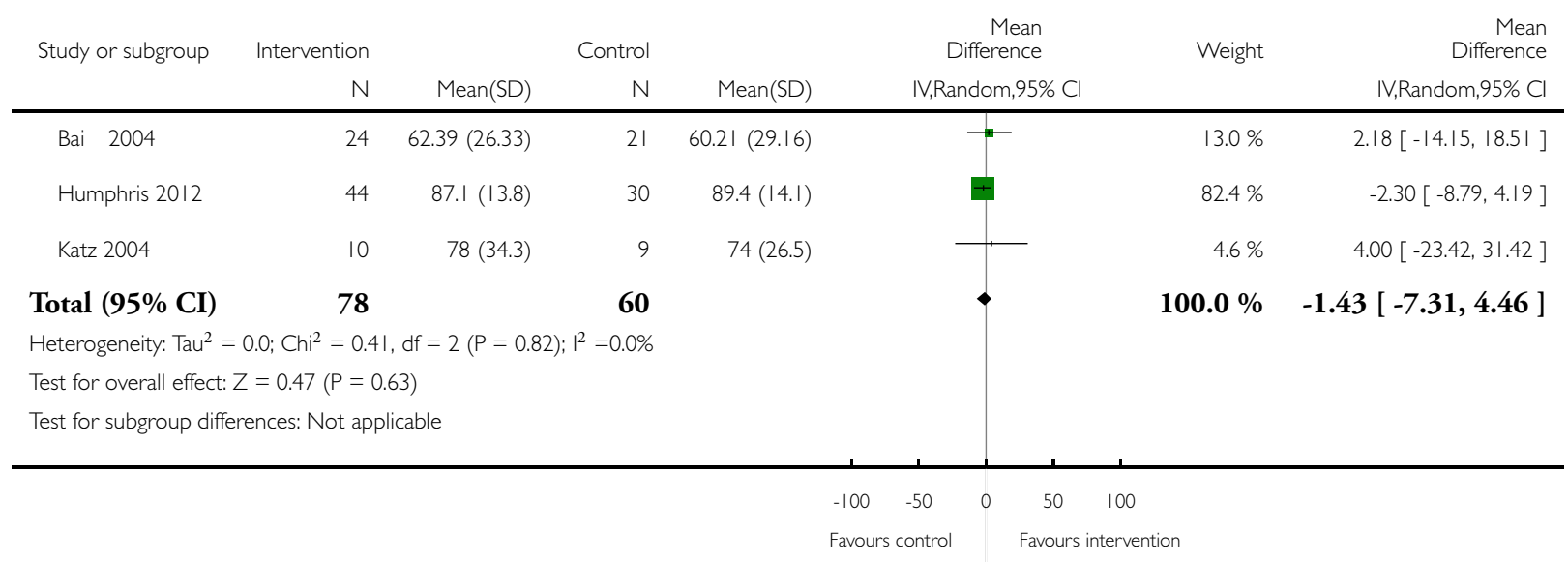


Analysis I.5. Comparison I Intervention versus control, Outcome 5 EORTC QLQ C30 - Global quality of life (medium-term follow-up).

Review: Psychosocial interventions for patients with head and neck cancer

Comparison: I Intervention versus control

Outcome: 5 EORTC QLQ C30 - Global quality of life (medium-term follow-up)

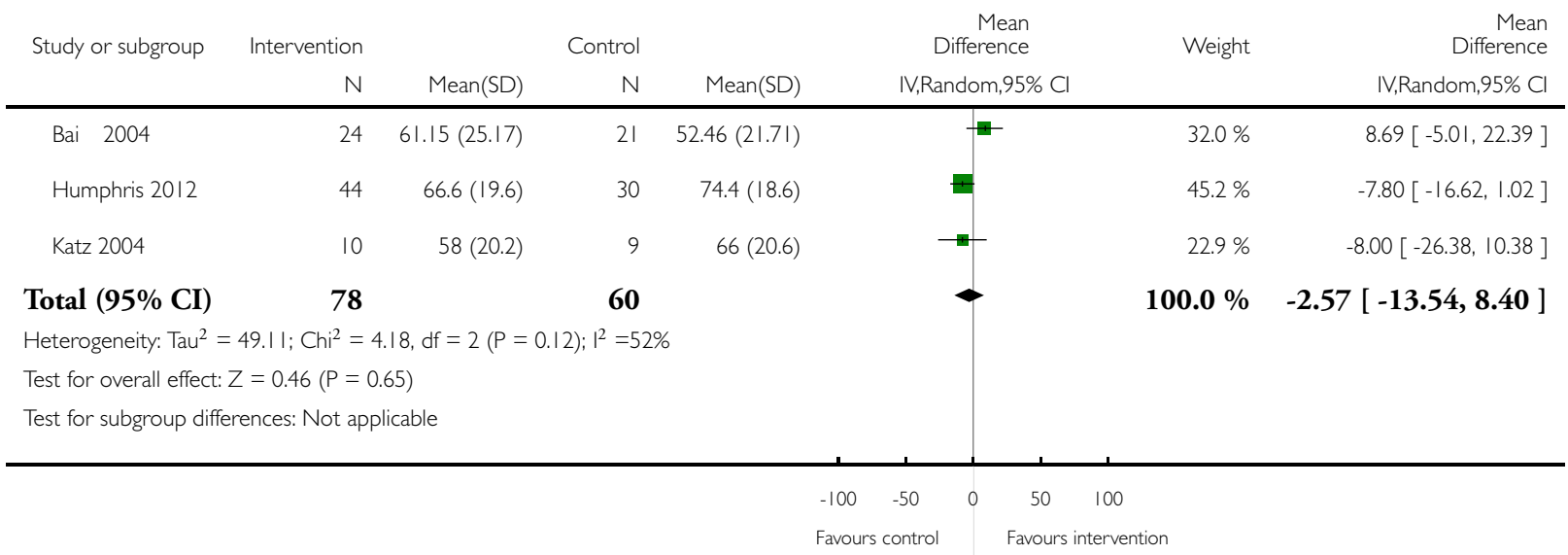


Analysis I.6. Comparison I Intervention versus control, Outcome 6 EORTC QLQ C30 - Cognitive function (medium-term follow-up).

Review: Psychosocial interventions for patients with head and neck cancer

Comparison: I Intervention versus control

Outcome: 6 EORTC QLQ C30 - Cognitive function (medium-term follow-up)

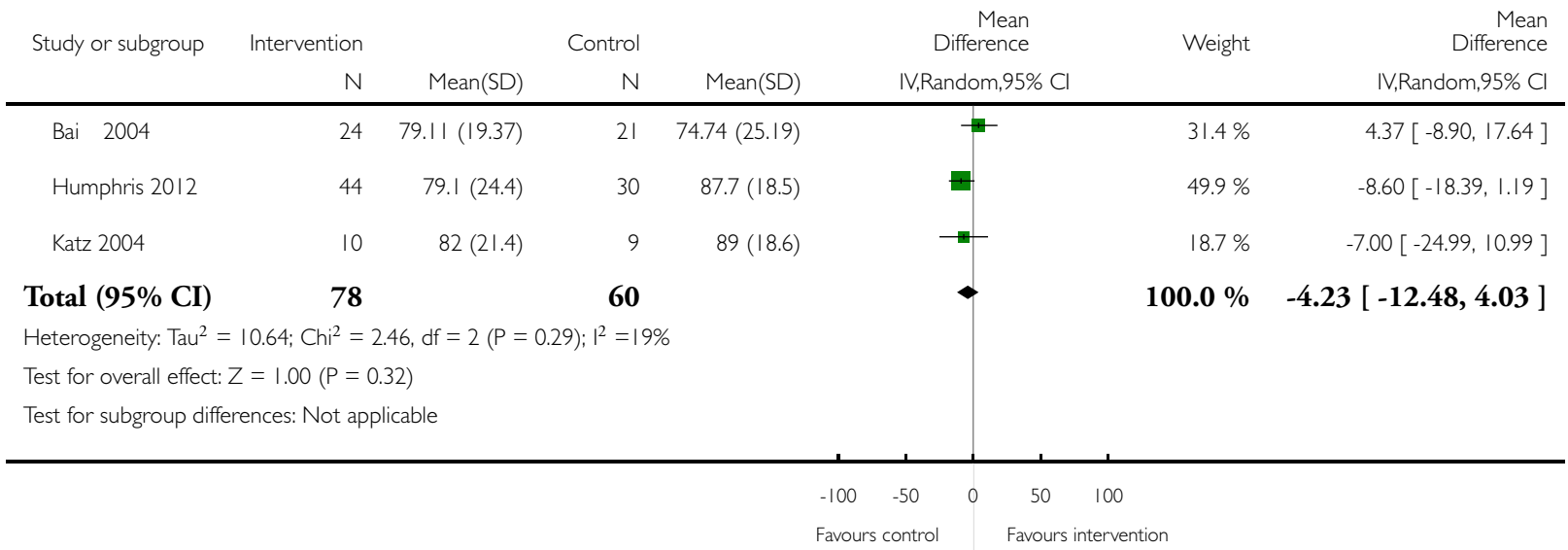


Analysis I.7. Comparison I Intervention versus control, Outcome 7 EORTC QLQ C30 - Cognitive function (end of intervention).

Review: Psychosocial interventions for patients with head and neck cancer
Comparison: I Intervention versus control
Outcome: 7 EORTC QLQ C30 - Cognitive function (end of intervention)

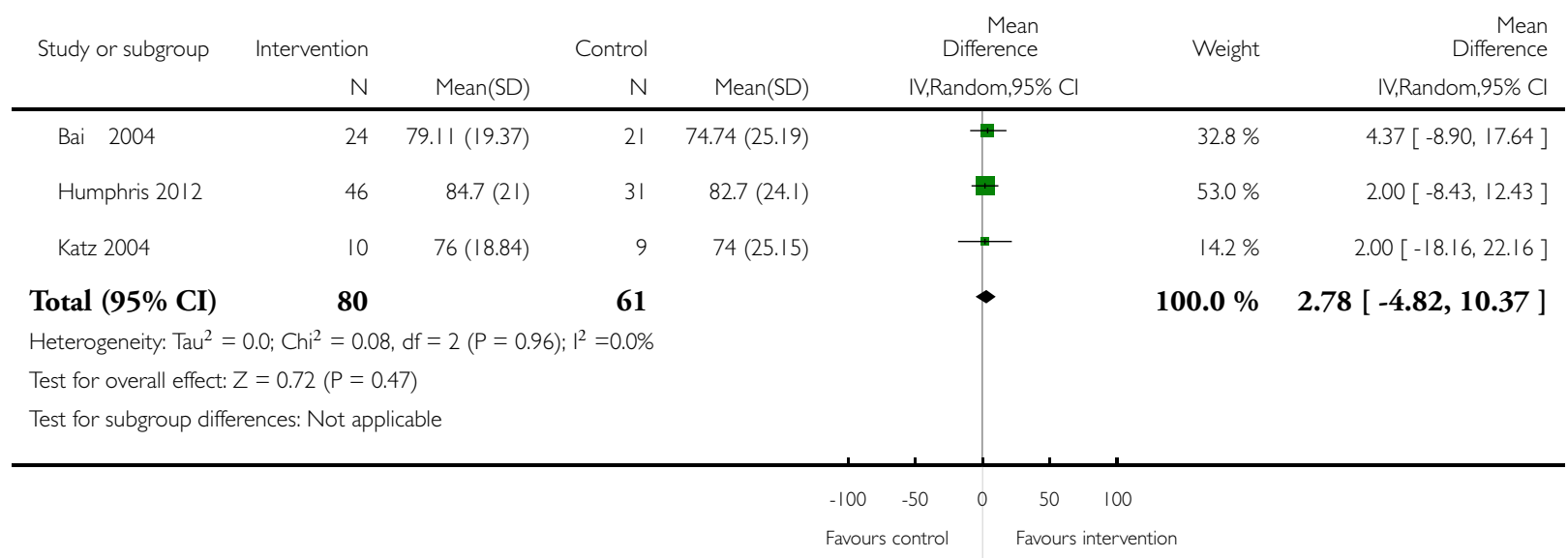


Analysis I.8. Comparison I Intervention versus control, Outcome 8 EORTC QLQ C30 - Physical function (end of intervention).

Review: Psychosocial interventions for patients with head and neck cancer

Comparison: I Intervention versus control

Outcome: 8 EORTC QLQ C30 - Physical function (end of intervention)

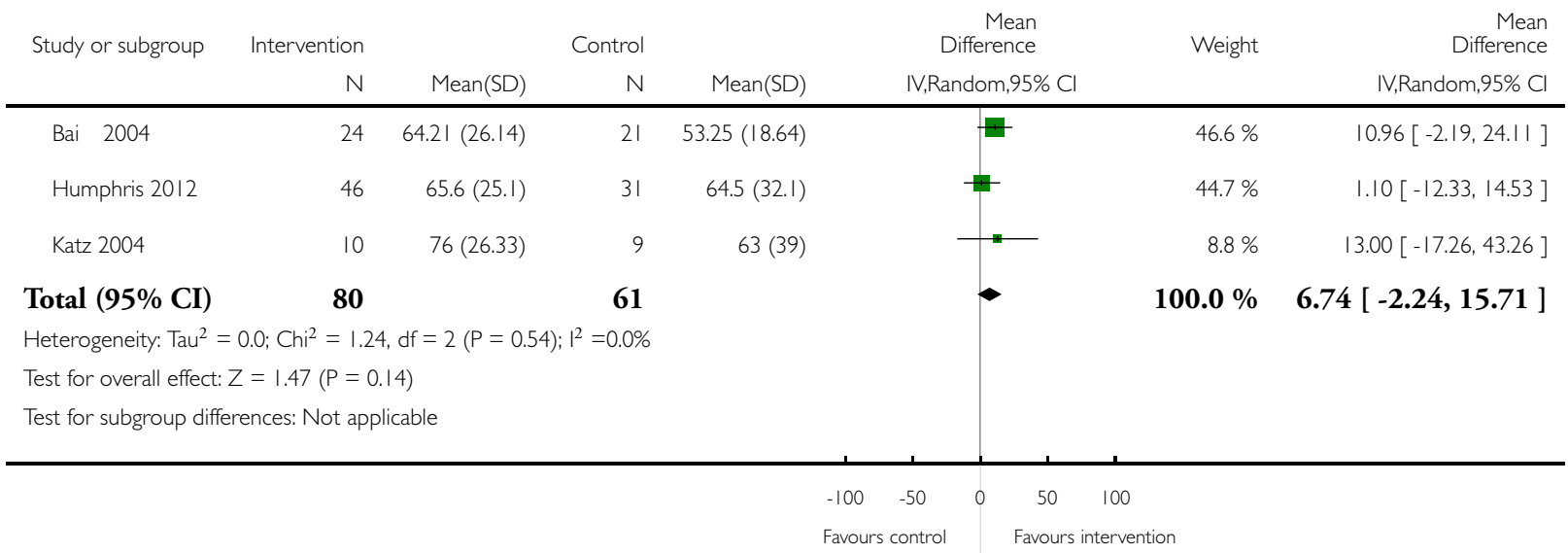


Analysis I.9. Comparison I Intervention versus control, Outcome 9 EORTC QLQ C30 - Emotional function (end of intervention).

Review: Psychosocial interventions for patients with head and neck cancer
Comparison: I Intervention versus control
Outcome: 9 EORTC QLQ C30 - Emotional function (end of intervention)

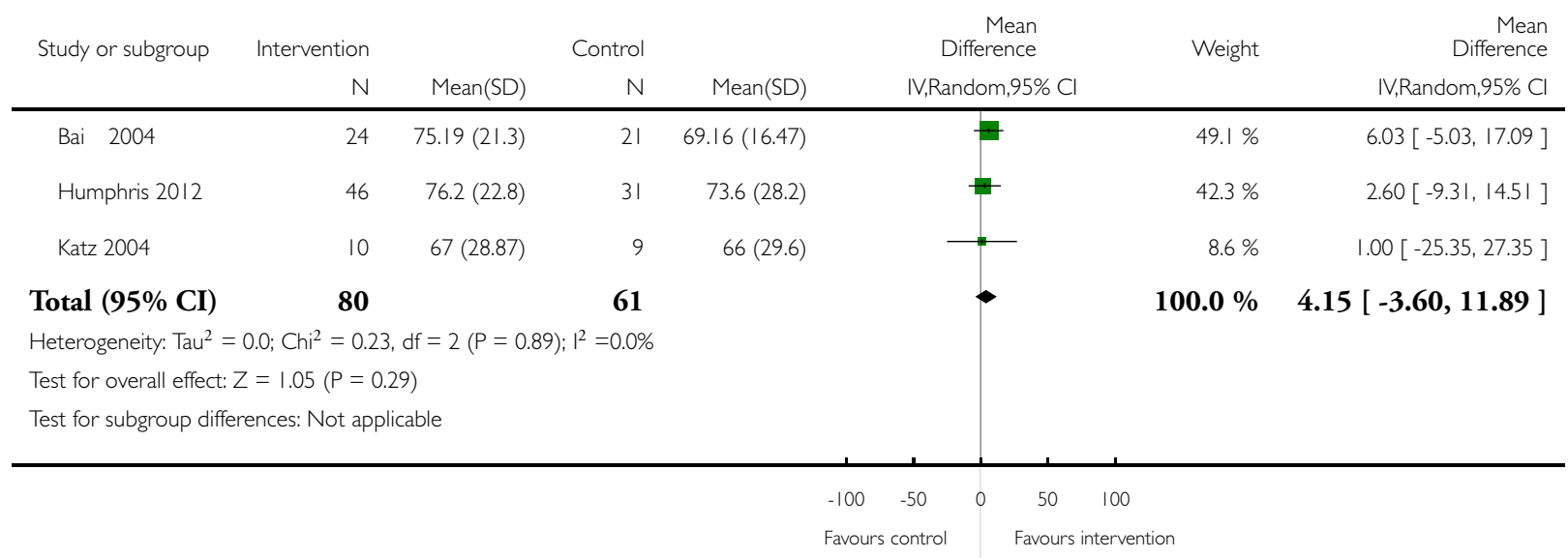


Analysis I.I0. Comparison I Intervention versus control, Outcome I0 EORTC QLQ C30 - Social function (end of intervention).

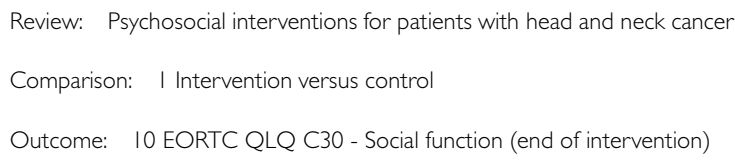

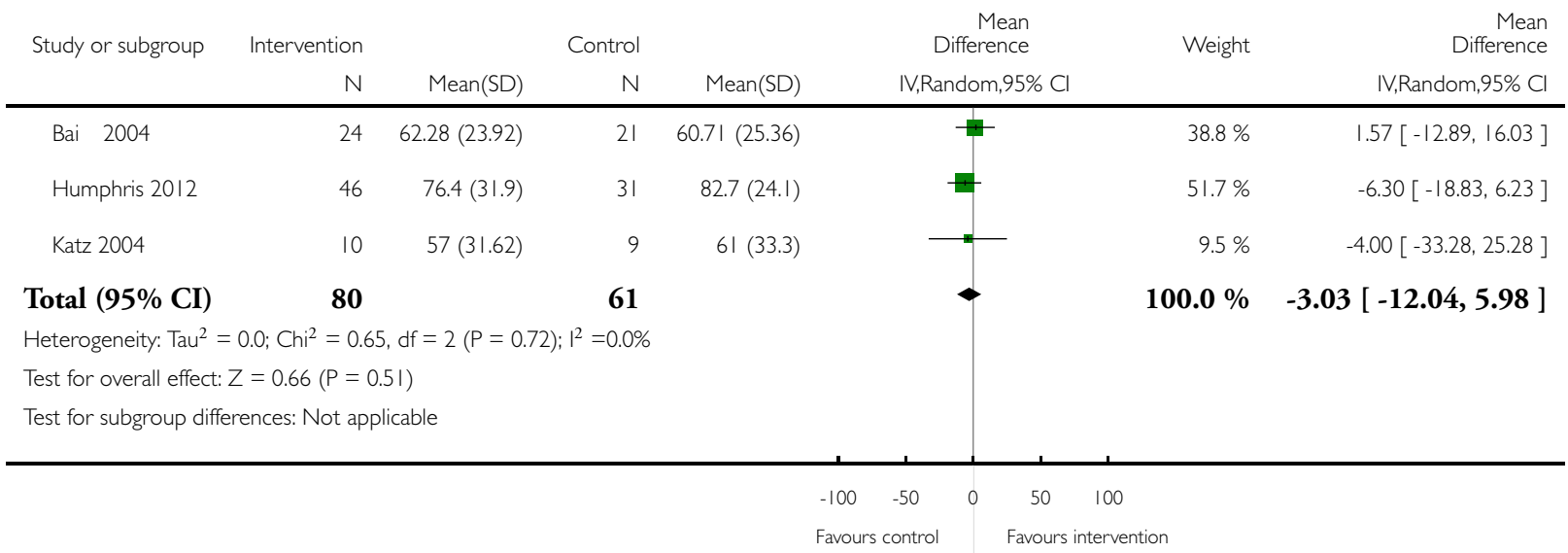


Analysis I.I I. Comparison I Intervention versus control, Outcome I I EORTC QLQ C30 - Role function (end of intervention).

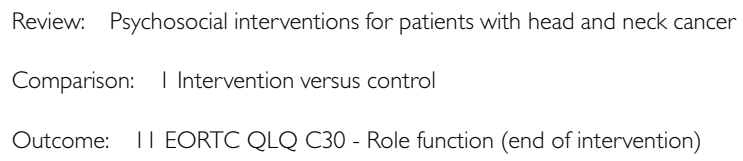

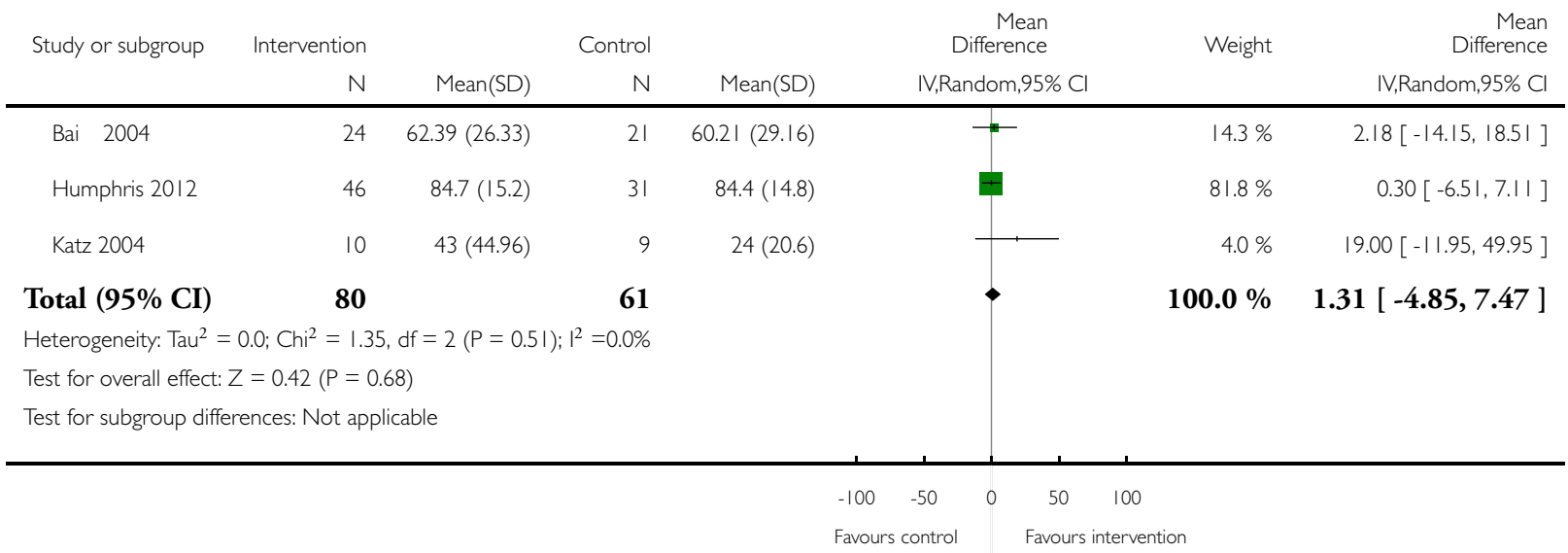


Analysis I.12. Comparison I Intervention versus control, Outcome I2 EORTC QLQ C30 - Global quality of life (end of intervention).

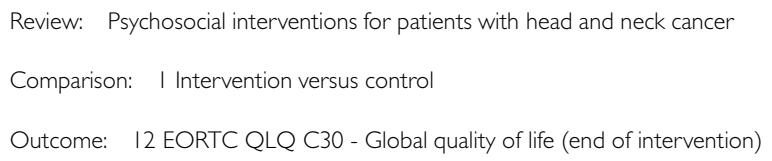

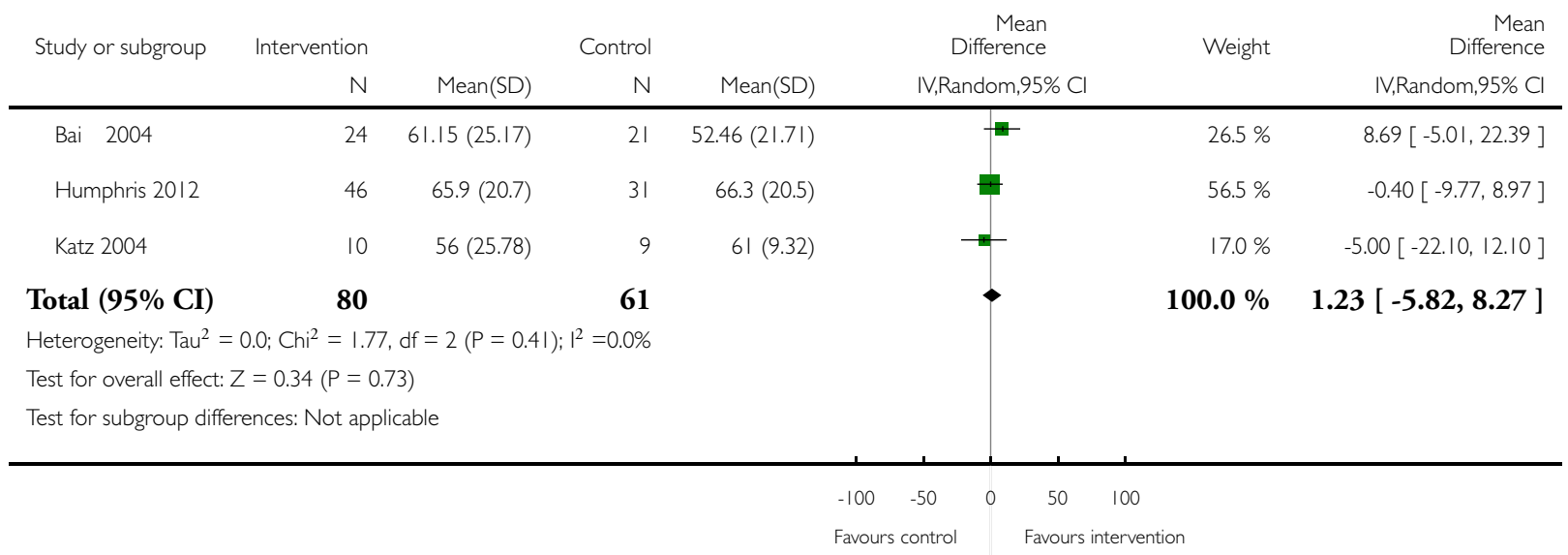


Analysis I.13. Comparison I Intervention versus control, Outcome I3 Anxiety (medium-term follow-up 3 to 4 months).

Review: Psychosocial interventions for patients with head and neck cancer

Comparison: I Intervention versus control

Outcome: 13 Anxiety (medium-term follow-up 3 to 4 months)

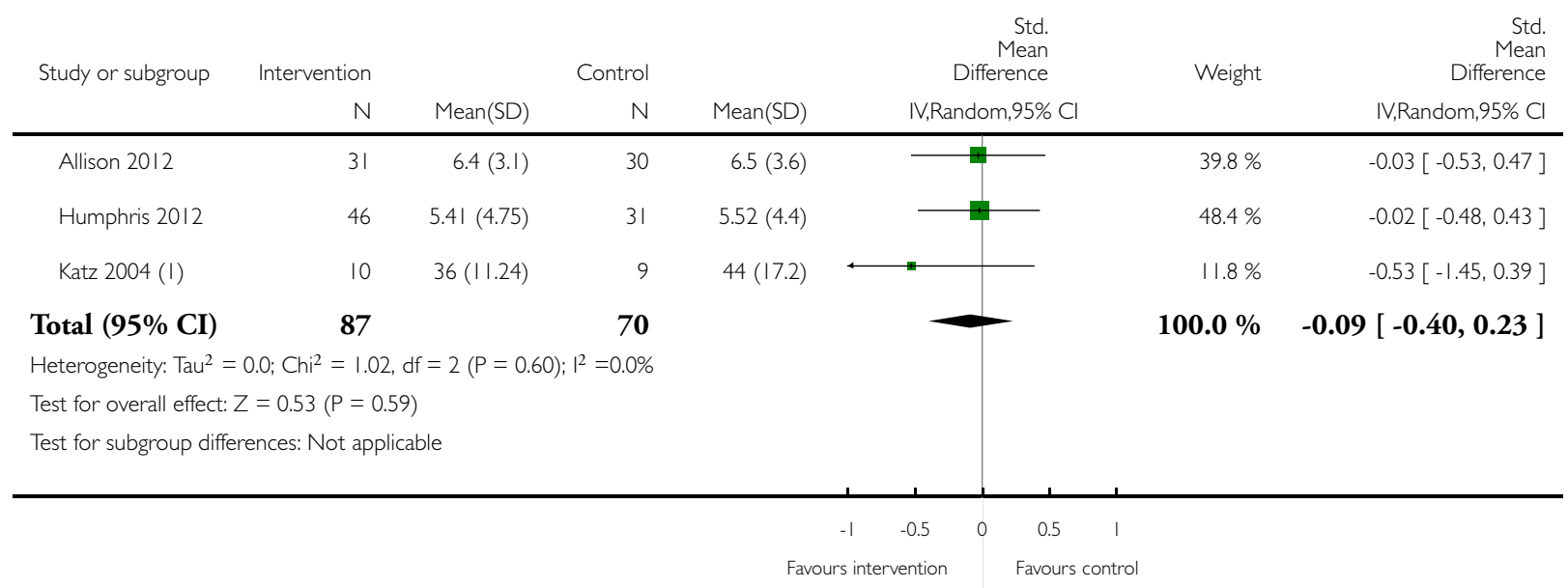

( I) STAl-state 


\section{Analysis I.14. Comparison I Intervention versus control, Outcome I4 Anxiety (end of intervention).}

Review: Psychosocial interventions for patients with head and neck cancer

Comparison: I Intervention versus control

Outcome: 14 Anxiety (end of intervention)

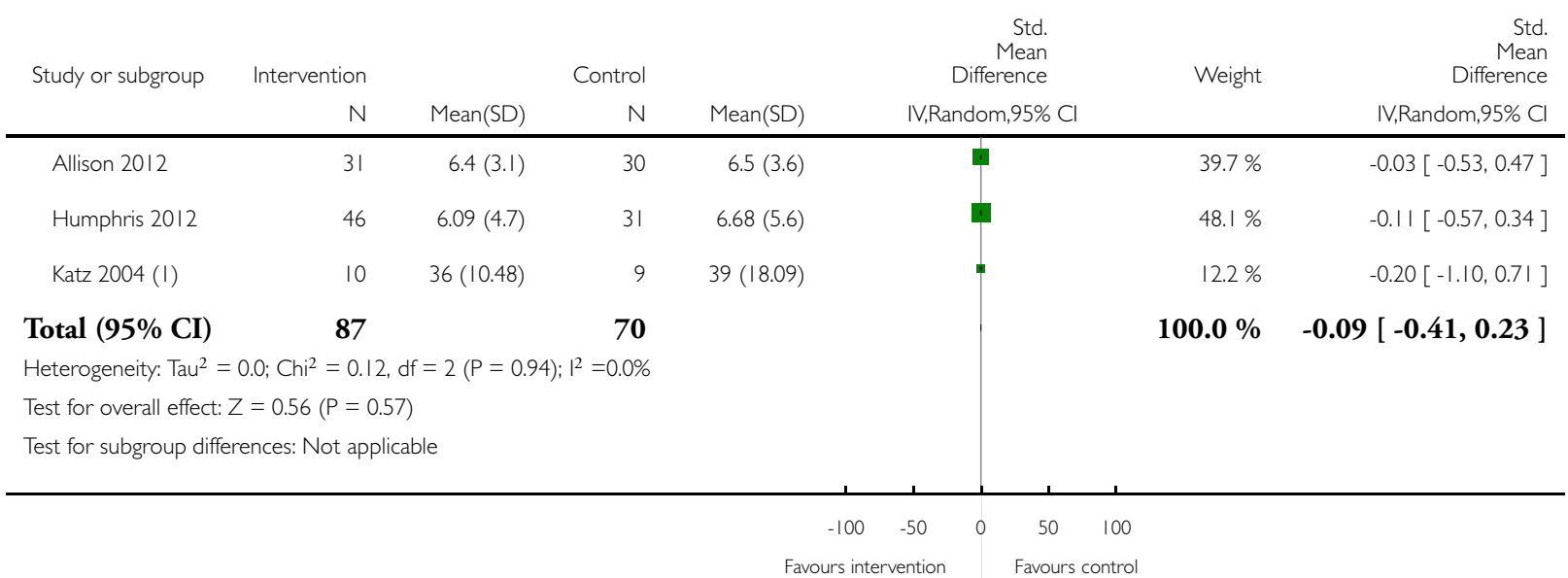

( I) (I) STAl-state 
Analysis I.I5. Comparison I Intervention versus control, Outcome I5 Depression (medium term follow-up 3 to 6 months).

Review: Psychosocial interventions for patients with head and neck cancer

Comparison: I Intervention versus control

Outcome: 15 Depression (medium term follow-up 3 to 6 months)

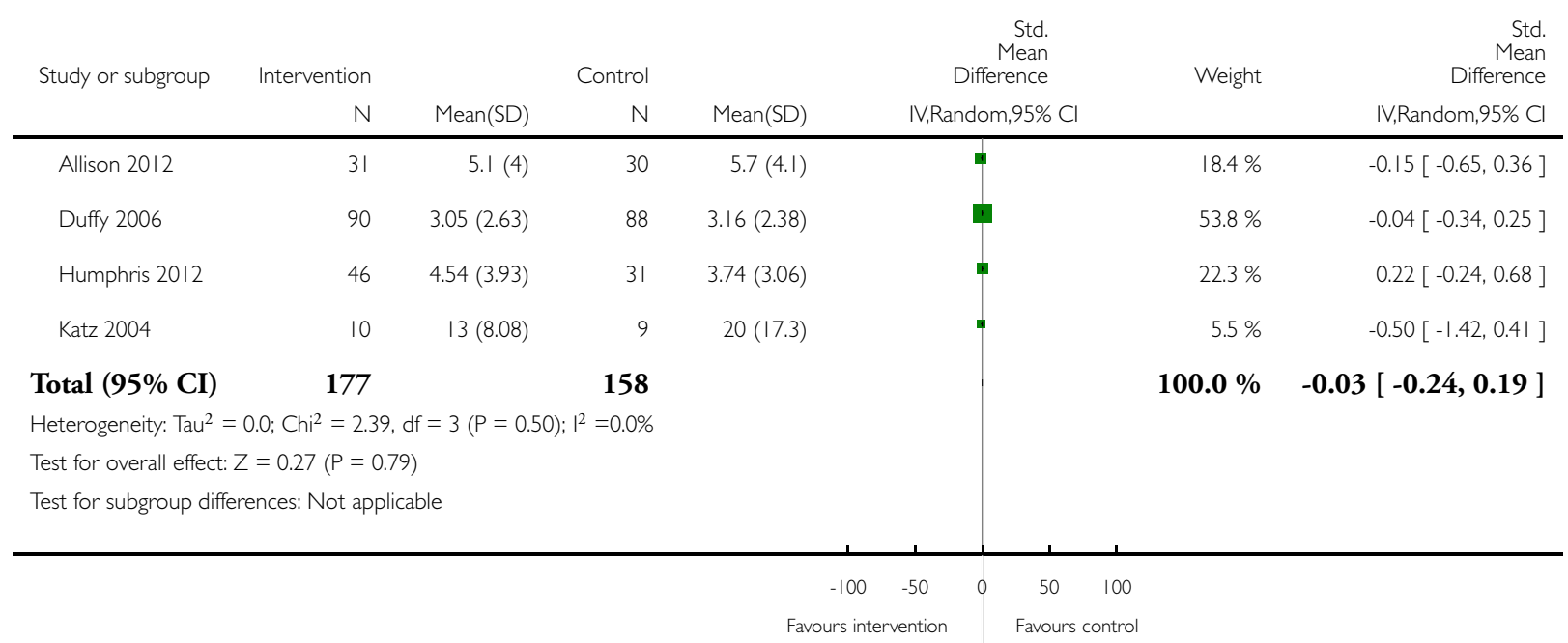


Analysis I.16. Comparison I Intervention versus control, Outcome I6 Depression (end of intervention).

Review: Psychosocial interventions for patients with head and neck cancer

Comparison: I Intervention versus control

Outcome: 16 Depression (end of intervention)

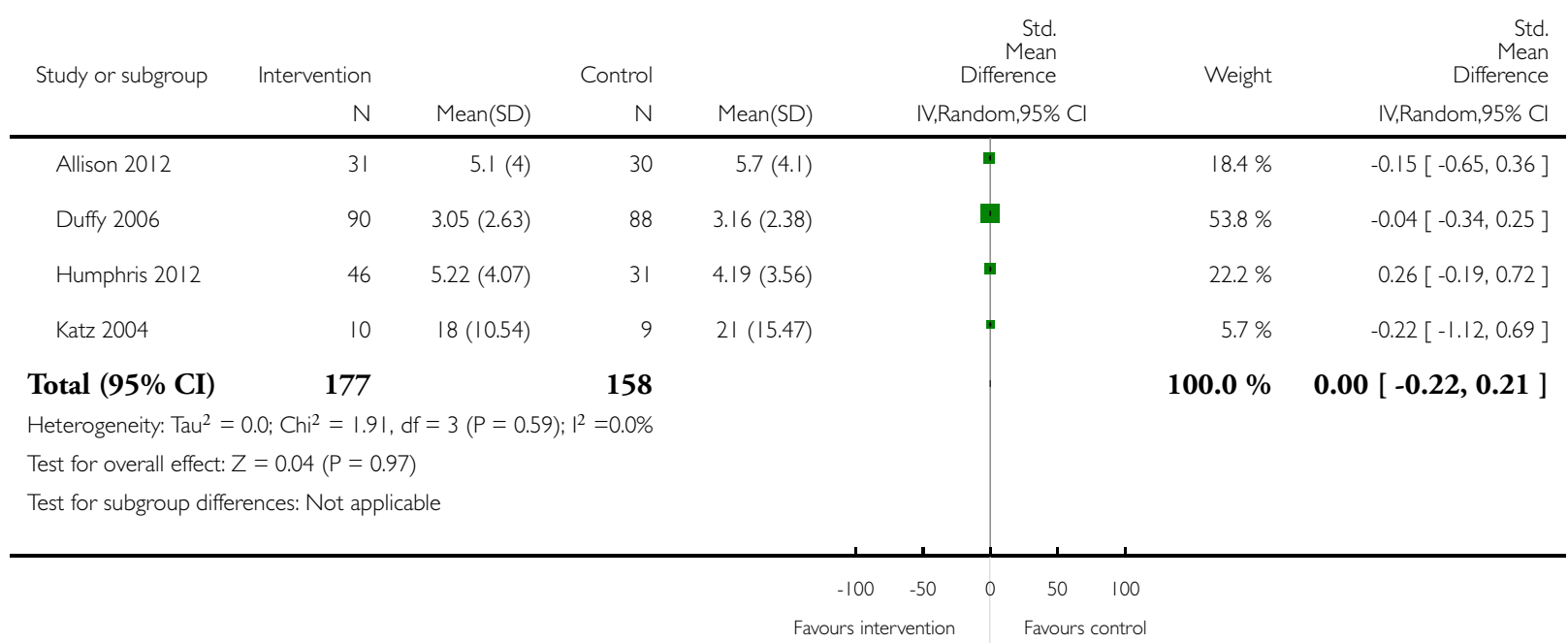

A P P E N I C ES

\section{Appendix I. Search strategies}

\begin{tabular}{|c|c|c|c|}
\hline CENTRAL & $\begin{array}{l}\text { Cochrane ENT Disorders } \\
\text { Group Trials Register }\end{array}$ & PubMed & EMBASE (Ovid) \\
\hline $\begin{array}{l}\text { \#1 MeSH descriptor Head and } \\
\text { Neck Neoplasms, this term only } \\
\text { \#2 MeSH descriptor Otorhi- } \\
\text { nolaryngologic Neoplasms ex- } \\
\text { plode all trees } \\
\text { \#3 MeSH descriptor Neck Dis- } \\
\text { section explode all trees } \\
\text { \#4 MeSH descriptor Laryngec- } \\
\text { tomy explode all trees } \\
\text { \#5 MeSH descriptor Neo- } \\
\text { plasms explode all trees }\end{array}$ & $\begin{array}{l}\text { (HNSCC OR SCCHN OR la- } \\
\text { ryngectom* OR (neck AND } \\
\text { dissect*) OR cancer* OR car- } \\
\text { cinoma* OR neoplas* OR tu- }^{*} \text { mor* OR tumour* OR malig- }^{*} \text { nan* OR SCCA) AND (coun- } \\
\text { sel* OR psychoeducat* OR ed- }^{*} \text { ucat* OR psychological OR } \\
\text { coping OR psychotherap* OR } \\
\text { psychosocial* OR psychoana- }\end{array}$ & $\begin{array}{l}\# 1 \text { "Head and Neck Neo- } \\
\text { plasms"[Mesh:NoExp] } \\
\# \\
2 \text { "Otorhinolaryngologic Neo- } \\
\text { plasms" [Mesh] OR "Neck Dis- } \\
\text { section" [Mesh] OR "Laryngec- } \\
\text { tomy" [MeSH] OR HNSCC } \\
\text { OR SCCHN OR laryngectom* } \\
\text { OR (neck AND dissect*) } \\
\text { \#3 "Neoplasms" [Mesh] OR }\end{array}$ & $\begin{array}{l}1 \text { exp "head and neck cancer"/ } \\
2 \text { exp neck dissection/ } \\
3 \text { exp laryngectomy/ } \\
4 \text { (HNSCC or SCCHN or la- } \\
\text { ryngectom* or (neck and dis- } \\
\text { sect*)).tw. } \\
5 \text { exp neoplasm/ } \\
6 \text { (cancer or cancers or cancer- } \\
\text { ous or carcinoma* or neoplas* } \\
\text { or tumor* or tumour* or malig- }\end{array}$ \\
\hline
\end{tabular}

Psychosocial interventions for patients with head and neck cancer (Review)

Copyright $\odot 2013$ The Cochrane Collaboration. Published by John Wiley \& Sons, Ltd. 
\#6 (cancer* OR carcinoma* OR neoplas* OR tumor* OR tumour* OR malignan* OR SCCA)

\#7

("head and neck":ti OR "head neck":ti OR "head-neck":ti OR "head-and-neck":ti OR oral: ti OR oropharyn*:ti OR hypopharyn*:ti or laryn*:ti OR nasopharyn*:ti OR pharyn*:ti OR throat:ti OR mouth:ti)

\#8 (\#5 OR \#6) AND \#7

\#9 HNSCC OR SCCHN OR

laryngectom* OR (neck AND

dissect*)

\#10 \#1 OR \#2 OR \#3 OR \#4 OR \#8 OR \#9

\#11 MeSH descriptor Counseling explode all trees

\#12 MeSH descriptor Psychotherapy explode all trees

\#13 MeSH descriptor SelfHelp Groups explode all trees \#14 MeSH descriptor MindBody Therapies explode all trees \#15 MeSH descriptor Religion and Psychology explode all trees \#16 MeSH descriptor Patient Education as Topic explode all trees

\#17 MeSH descriptor Personal Satisfaction explode all trees

\#18 MeSH descriptor Mental Health explode all trees

\#19 MeSH descriptor Quality of Life explode all trees with qualifier: PX

\#20 MeSH descriptor Emotions explode all trees with qualifier: $\mathrm{TH}$

\#21 MeSH descriptor Stress, Psychological explode all trees with qualifier: $\mathrm{TH}$

\#22 MeSH descriptor Depression explode all trees with qualifier: $\mathrm{TH}$

\#23 counsel*:ti OR psychoeducat*:ti OR coping:ti OR lytic OR social OR peer OR help OR cognitive OR relax* OR family OR couple OR bibliotherap* OR art OR music OR colour OR color OR relig* OR prayer* OR spiritual* OR meditat* OR aromatherap*)

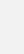

(cancer OR cancers OR cancerous OR carcinoma* OR neoplas* OR tumor* OR tumour* OR malignan* OR SCCA) \#4 ("head and neck" [ti] OR "head neck" [ti] OR "headneck" [ti] OR "head-and-neck" [ti] OR oral [ti] OR oropharyn* [ti] OR hypopharyn* [ti] or laryn* [ti] OR nasopharyn* [ti] OR pharyn* [ti] OR throat [ti] OR mouth [ti])

\#5 \#3 AND \#4 \#6 \#1 OR \#2 OR \#5

\#7 "Counseling” [Mesh] OR "Psychotherapy" [Mesh] OR "Self-Help Groups" [Mesh] OR "Mind-Body Therapies" [Mesh] OR "Religion and Psychology” [Mesh] OR "Personal Satisfaction" [MeSH] OR "Mental Health" [Mesh] OR "Quality of Life/psychology" [Mesh] OR "Emotions/therapy" [Mesh] OR "Stress, Psychological/therapy" [Mesh] OR "Depression/therapy" [Mesh] \#8 "Patient Education as Topic"[Mesh]

\#9 counsel* $^{*}$ [ti] OR psychoeducat* [ti] OR educat* [ti] OR coping [ti] OR (psychological [ti] AND (therap* [ti] OR treatment* [ti] OR outcome* $^{*}$ [ti] OR intervention* [ti])) OR psychotherap* [ti] OR

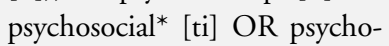
analytic [ti] OR ((social [ti] OR peer [ti] OR group [ti]) AND support [ti]) OR (self [ti] AND help [ti]) OR (cognitive [ti] AND (therap* [ti] OR behav* [ti])) OR relax* [ti] OR ((family [ti] OR couple [ti]) AND therap* [ti]) \#10 bibliotherap* [ti] OR ((art [ti] OR music [ti] OR colour [ti] OR color [ti]) AND therap* nan* or SCCA).tw.

75 or 6

8 ((head and neck) or oral or oropharyn* or hypopharyn*

or laryn* or nasopharyn* or pharyn* or throat or mouth).tw. 97 and 8

101 or 2 or 3 or 4 or 9

11 exp psychotherapy/

12 animal assisted therapy/ or counseling/

13 exp self help/

14 exp mental health/

15 exp religion/

16 [exp emotion/th [Therapy]]

17 [exp stress/th [Therapy]]

18 [exp depression/th [Ther-

apy]]

19 (counsel* $^{*}$ or psychoeducat* or educat* or coping or (psychological and (therap* or treatment* or outcome* or intervention*)) or psychotherap* or psychosocial* or psychoanalytic or ((social or peer or group) and support) or (self and help) or (cognitive and (therap* or behav $\left.^{*}\right)$ ) or relax* or ((family or couple) and therap*) or bibliotherap* or ((art or music or colour or color) and therap*) or relig* or prayer* or spiritual* or meditat* or aromatherap*).ti. 20 exp aromatherapy/ or exp reiki/ or exp spiritual healing/ 2111 or 12 or 13 or 14 or 15 or 16 or 17 or 18 or 19 or 20 2210 and 21

Psychosocial interventions for patients with head and neck cancer (Review) 
educat*:ti OR (psychological: ti AND (therap*:ti OR treatment*:ti

OR

outcome*:ti OR intervention*: ti)) OR psychotherap*:ti OR psychosocial*:ti OR psychoanalytic:ti OR ((social:ti OR peer: ti OR group:ti) AND support: ti) OR (self:ti AND help:ti) OR (cognitive:ti AND (therap*:ti OR behav*:ti)) OR relax*:ti OR ((family:ti OR couple:ti) AND therap*:ti)

\#24 bibliotherap*:ti OR ((art:ti OR music:ti OR colour:ti OR color:ti) AND therap*:ti) OR relig*:ti OR prayer*:ti OR spiritual ${ }^{*}$ :ti OR meditat*:ti OR aromatherap*:ti

\#25 \#11 OR \#12 OR \#13 OR

\#14 OR \#15 OR \#16 OR \#17

OR \#18 OR \#19 OR \#20 OR

\#21 OR \#22 OR \#23 OR \#24

\#26 \#10 AND \#25 [ti]) OR relig* [ti] OR prayer* [ti] OR spiritual* [ti] OR meditat* [ti] OR aromatherap* [ti] \#11 \#7 OR \#8 OR \#9 OR \#10 \#12 \#6 AND \#11

\section{Web of Science/BIOSIS Pre- CINAHL (EBSCO) views (Web of Knowledge)}

\#1 TS=(HNSCC OR SCCHN OR laryngectom* OR (neck AND dissect*))

\#2 TS $=$ (cancer ${ }^{*}$ OR carcinoma* OR neoplas* OR tumor* OR tumour* OR malignan* OR SCCA)

\#3 TS=((head and neck) or oral or oropharyn* or hypopharyn* or laryn* or nasopharyn* or pharyn* or throat or mouth) \#4 \#3 AND \#2

\#5 \#4 OR \#1

\#6 TI=(counsel* OR psychoeducat* OR educat* OR (psychological AND (therap* OR treatment*)) OR psychotherap* OR psychosocial* OR psychoanalytic OR ((social OR peer OR group) AND support) OR (self AND help) OR (cogni-
S1 (MH "Head and Neck Neo- 1 exp "head and neck cancer plasms+") OR (MH "Otorhi- 2 exp laryngeal cancer nolaryngology and Head-Neck Nursing")

S2 (MH "Laryngectomy")

3 (HNSCC or SCCHN or laryngectom* or (neck and dissect $\left.\left.{ }^{*}\right)\right)$.tw.

S3 TX (HNSCC OR SCCHN 4 exp neoplasm/

OR laryngectom* OR (neck AND dissect*))

S4 S1 or S2 or S3

S5 (MH "Neoplasms+")

S6 TX (cancer* OR carcinoma* OR neoplas* OR tumor* OR tumour* OR malignan* ${ }^{*}$ OR

SCCA)

S7 S5 or S6

S8 TX ((head and neck) or oral or oropharyn* or hypopharyn* or laryn* or nasopharyn* or pharyn* or throat or mouth)

S9 S7 and S8

S10 S4 or S9
5 (cancer or cancers or cancerous or carcinoma* or neoplas* or tumor* or tumour* or malignan* or SCCA).tw.

64 or 5

7 ((head and neck) or oral or oropharyn* or hypopharyn* or laryn* or nasopharyn* or pharyn* or throat or mouth).tw. 86 and 7

91 or 2 or 3 or 8

10 exp psychotherapy/

11 exp counseling/

12 exp self help/

13 exp mental health/ head AND neck AND psych* OR head AND neck AND

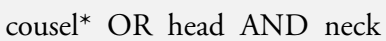
AND coping OR head AND neck AND stress* OR head AND neck AND depression OR hnscc AND psych* OR hnscc AND counsel* OR hnscc AND stress* OR hnscc AND depress OR hnscc AND coping

Psychosocial interventions for patients with head and neck cancer (Review)

Copyright @ 2013 The Cochrane Collaboration. Published by John Wiley \& Sons, Ltd. 
tive AND (therap* OR behav*) ) OR relax* OR ((family OR couple) AND therap*) OR bibliotherap* OR ((art OR music OR colour OR color) AND therap*) OR relig* OR prayer* OR spiritual* OR meditat* OR aromatherap*)

\#7 \#6 AND \#5
S11 (MH "Psychotherapy+")

OR (MH "Bibliotherapy")

S12 (MH "Counseling+")

S13 (MH "Support Groups+")

S14 (MH "Patient Education+")

S15 (MH "Mental Health")

S16 (MH "Religion and Psychology+")

S17 (MH "Quality of Life/PF")

S18 (MH "Stress/TH")

S19 (MH "Depression/TH”)

S20 (MH "Disease/PF")

S21 TI (counsel* OR psychoeducat* OR educat* OR (psychological AND (therap* OR treatment*)) OR psychotherap* OR psychosocial* $^{*}$ OR psychoanalytic OR ((social OR peer OR group) AND support) OR (self AND help) OR (cognitive AND (therap* OR behav*) ) OR relax* OR ((family OR couple) AND therap*) OR bibliotherap* OR ((art OR music OR colour OR color) AND therap*) OR relig* OR prayer* OR spiritual* OR meditat* OR aromatherap*)

S22 S11 or S12 or S13 or S14 or $S 15$ or $S 16$ or $S 17$ or $S 18$ or S19 or S20 or S21

S23 S10 and S22
14 exp religion/

15 (counsel* $^{*}$ or psychoeducat* or educat* or coping or (psychological and (therap* or treatment* or outcome* or intervention*)) or psychotherap* or psychosocial* or psychoanalytic or ((social or peer or group) and support) or (self and help) or (cognitive and (therap* or behav $\left.^{*}\right)$ ) or relax* or ((family or couple) and therap*) or bibliotherap* or ((art or music or colour or color) and therap*) or relig* or prayer* or spiritual* or meditat* or aromatherap*).ti. 16 exp aromatherapy/ 1710 or 11 or 12 or 13 or 14 or 15 or 16

189 and 17

\section{CONTRIBUTIONSOFAUTHORS}

CS was the main author and was involved in all aspects of the review.

$\mathrm{KP}$ provided supervision, appraised papers, extracted data and commented on the drafts.

AN appraised papers, provided statistical advice and commented on the drafts.

$\mathrm{EMcC}$ appraised papers, extracted data and commented on the drafts.

MM appraised papers and commented on the drafts.

$\mathrm{GH}$ provided editorial assistance with the review.

Psychosocial interventions for patients with head and neck cancer (Review) 


\section{DECLARATIONSOF INTEREST}

G Humphris has received an honorarium from Merck Serono Ltd for his presentation on quality of life in head and neck cancer patients to a group of Irish head and neck cancer specialists in Dublin in 2009. He is also the primary author of one of the included studies in this review (Humphris 2012).

All other authors: none known.

\section{SOURCES OF SUPPORT}

\section{Internal sources}

- No sources of support supplied

\section{External sources}

- Health Research Board/HSC R\&D, Cochrane Fellowship Award, UK.

\section{DIFFERENCES BETWEEN PROTOCOLANDREVIEW}

Due to the small number of studies and the methodological quality of the available studies, we did not perform subgroup analysis. 